\title{
A REVIEW OF RADIO INTERFEROMETRIC IMAGING OF COMETS
}

\author{
Imke de Pater* \\ Astronomy Department \\ 601 Campbell Hall. \\ University of California, Berkeley, CA 9/720 \\ Patrick Palmer \\ Department of Astronomy and Astrophysics \\ University of Chicago \\ 5640 South Ellis Ave. \\ Chicago, IL 60637 \\ Lewis E. Snyder \\ Department of Astronomy \\ University of Illinois \\ 349 Astronomy Bldg. \\ 1011 W. Springfield Ave. \\ Urbana, IL 61801
}

\begin{abstract}
We present an overview of cometary observations carried out with the VLA. These include $\mathrm{OH}$ imaging observations, studies of other molecules and searches for continuum emission. Primarily, we discuss the $\mathrm{OH}$ observations obtained of Comet Halley, a periodic comet, and Comet Wilson, a "new" comet. The emission from Comet Halley is confined to a region a few times $10^{5}$ $\mathrm{km}$. The emission from Comet Wilson shows sporadic blobs at large distances $\left(\sim 10^{6} \mathrm{~km}\right.$ from the center), which vary abruptly in both position and velocity. The generally accepted picture of excitation by UV pumping in a spherical outflow of cometary gas explains the coarse phenomenology, but other effects seem to cause significant modulations of the $\mathrm{OH}$ emission. We suggest that for Comet Halley, collisional effects near the edge of the quenching region, which is coincidently the approximate distance to the cometopause, may be important, and that for Comet Wilson, outgassing from cometesimals ejected from the nucleus may be significant. We further show that asymmetric line profiles are more common than previously thought. Lengthening integration times and lowering the velocity resolution results in a smooth, round shape for the comet's brightness distribution. In addition, spectral profiles become broader and more symmetric after long integration times and after integration over larger spatial regions. In addition to the $\mathrm{OH}$ observations we summarize the $\mathrm{H}_{2} \mathrm{CO}$ detection experiments for comets Halley and Machholz.
\end{abstract}

\section{Introduction}

It is generally accepted that water ice is the primary constituent of a cometary nucleus and sublimated $\mathrm{H}_{2} \mathrm{O}$ flows radially away as the comet approaches the sun. It is very difficult to observe $\mathrm{H}_{2} \mathrm{O}$ directly from the ground. Two probable detections have been made at $22 \mathrm{GHz}$ (Jackson, Clark and Donn, 1976; Altenhoff et al., 1983). Snyder (1982) has summarized a

\footnotetext{
*Alfred P. Sloan Fellow.
} 
number of searches for $\mathrm{H}_{2} \mathrm{O}$ and other molecules at radio wavelengths. Combes et al. (1986) identified fluorescence signatures of $\mathrm{H}_{2} \mathrm{O}$ with the IKS experiment on board VEGA 1 . The first clear "ground-based" detection of water vapor in a comet was obtained at infrared wavelengths from the Kuiper Airborne Observatory (Weaver, Mumma, and Larson, 1987).

After an exponential lifetime of $\sim 10^{5} \mathrm{sec}$, solar ultraviolet light will photodissociate $60-90 \%$ of the $\mathrm{H}_{2} \mathrm{O}$ into $\mathrm{OH}+\mathrm{H}$ (Festou, 1981a,b; Schloerb and Gerard, 1985; Snyder, 1986 and references in these papers). It is relatively straightforward to observe the $\mathrm{OH}$ radical at both UV and radio wavelengths. Because $\mathrm{OH}$ is the most abundant easily observable molecule, its study provides the most direct information about the outgassing of a comet (see also Crovisier and Schloerb, 1990).

In order to derive information on the origin of comets, it is necessary to investigate the composition of the nucleus. Since one cannot observe this composition directly, the best one can do is to observe parent molecules in the cometary coma. Many potential parents (e.g., $\mathrm{HCN}, \mathrm{H}_{2} \mathrm{CO}, \mathrm{NH}_{3}$ ) have easily excited rotational transitions at radio wavelengths.

In this paper we will primarily discuss interferometric observations of cometary $\mathrm{OH}$ emission lines. In Section 2 the characteristics of $\mathrm{OH}$ emission/absorption are described. This section includes a general discussion of radio interferometry, and the results of some simulated cometary observations, which illustrate both the problems and the potential of this technique. In Section 3, the $\mathrm{OH}$ observations together with all other cometary observations made to date with the VLA (Very Large Array)* are summarized. In Sections 4 and 5, the results of the $\mathrm{OH}$ observations are discussed in more detail.

\section{Characteristics of $\mathrm{OH}$ Emission/Absorption}

$\mathrm{OH}$ lines from comets can be observed from the earth's surface at UV ( $\sim 3080$ Angstrom) and radio $(\sim 18 \mathrm{~cm})$ wavelengths. At radio wavelengths the lines are due to a lambda doubling transition and are seen either in absorption or maser emission. At UV wavelengths, the transitions are always seen in emission. In recent years UV studies of cometary OH have been carried out with the IUE satellite. It has been possible to image the $\mathrm{OH}$ emission at UV wavelengths, although with relatively poor velocity resolution $(\sim 25 \mathrm{~km} / \mathrm{sec})$. The images look smooth and symmetric (e.g., Ip, 1985). Palmer, de Pater and Snyder (1989) showed that while such smooth, symmetric images are seen when observing radio $\mathrm{OH}$ with low velocity resolution and/or long integration times, higher velocity resolution, shorter integration times and/or higher spatial resolution can show very irregular spatial and spectral patterns.

The $\mathrm{OH}$ molecules are excited from the hyperfine levels of the ground state by solar UV, and subsequently decay back to these hyperfine levels through a number of intermediate states. Since the excitation is caused by the Doppler shifted solar spectrum, the resulting relative populations of the the ground state hyperfine levels depend on the heliocentric velocity of the comet (or, more precisely, of the $\mathrm{OH}$ gas cloud surrounding the comet). If an upper level becomes overpopulated, the galactic background radiation causes maser emission of the corresponding $18 \mathrm{~cm} \mathrm{OH}$ line. If the comet's heliocentric velocity is such that a lower level becomes overpopulated, we will see the $\mathrm{OH}$ in absorption against the galactic background.

During a comet's perihelion passage, its heliocentric velocity changes drastically, and we see the cometary $\mathrm{OH}$ lines change from absorption to emission and back again. Using UV

*The VLA is operated by the National Radio Astronomy Observatory, which is operated by the Associated Universities, Inc., under contract with the National Science Foundation. 
pumping models (Despois et al., 1981; Schleicher and A'Hearn, 1988), one can predict the $\mathrm{OH}$ behavior of the comet as a function of heliocentric velocity (Swings effect). Galt (1987) showed a comparison of daily observations of the $\mathrm{OH}$ emission/absorption from Comet Halley with the behavior expected from the UV pumping models. There are two striking features in this body of data: 1) overall, the observed lines follow the predicted behavior, 2) on a day-to-day basis frequently there are deviations by a factor of 2 . This implies that the primary excitation mechanism of $\mathrm{OH}$ is indeed solar UV radiation. Deviations of the data from the model can be explained to first order by inclusion of the Greenstein effect, the differential motions of the gas inside the coma; but the variations on a daily basis require irregular gas production.

\subsection{LINE SHAPE}

The shape of the $\mathrm{OH}$ line implicitly contains information about the gas outflow from the comet. Excellent work on extracting information from such profiles has been carried out by the group at Meudon, e.g., Despois et al. (1981), Bockelee-Morvan and Gerard (1984). The early spectra (Despois et al., 1981) typically had a resolution of $1 \mathrm{~km} / \mathrm{sec}$ and were integrated over a couple of days. The full line width at half maximum intensity (FWHM) was typically $2-2.5 \mathrm{~km} / \mathrm{sec}$, implying an outflow velocity of about $1 \mathrm{~km} / \mathrm{sec}$. The profiles were generally roughly Gaussian in appearance, and often slightly skewed toward either the red or blue side. By comparison with the Haser model (uniform isotropic outgassing from the comet), these workers noted the presence of the Greenstein effect and sometimes needed to invoke anisotropic outgassing to fit the skewedness of the observed line profiles with a model.

In later years, the velocity resolution was improved by nearly an order of magnitude, to $0.14 \mathrm{~km} / \mathrm{sec}$ (Bockelee-Morvan and Gerard, 1984), which allowed the observers to derive conditions for the $\mathrm{OH}$ gas flow more precisely. They found that the gas flow could not be adequately represented by the Haser model. The $\mathrm{H}_{2} \mathrm{O}$ molecules have a bulk radial outflow velocity, but upon dissociation, the $\mathrm{OH}$ molecule is assumed to be ejected isotropically in the reference frame of the water molecule, with a small additional velocity. This could be modeled with a random walk model (Combi and Delsemme, 1980), or a vectorial model (Festou, 1981a). The results for the two computer methods are very similar (Festou, 1981a). Bockelee-Morvan and Gerard (1984) further included the quenching effect in their model: the suppression of maser emission in the inner dense coma, where the relative populations of the $\mathrm{OH}$ molecule are thermalized by collisions. They further noted that the line profiles often hinted at anisotropic outgassing. Unfortunately, the random walk and vectorial models have so many parameters that the models are not unique.

The effect of collisional quenching on the $\mathrm{OH}$ emission has been investigated quantitatively by Schloerb (1988). He finds that the $\mathrm{OH}$ production rates derived from radio and UV data can be reconciled if the same model is used for both and if quenching is taken into account.

\subsection{SPATIAL BRIGHTNESS DISTRIBUTION}

The spatial distribution of the $\mathrm{OH}$ emission can be measured accurately by making use of interferometers. With single antennas, crude maps were obtained with a resolution of a few arcminutes; with the VLA one can image the emission in detail. 
The basic principles of radio astronomy and interferometry have been described by e.g. Kraus (1986), Rohlfs (1986), Thompson et al. (1986), and Perley et al. (1989). The resolution for an interferometer pair is roughly $\lambda / D$, with $\lambda$ the observing wavelength and $D$ the projected antenna-pair separation. The measured quantities - correlation coefficients between antenna pairs - are related to the sky brightness by a Fourier transformation. A radio image can be constructed from observations obtained with many interferometer pairs at different spacings. It is important to note here that the resolution in an image is determined by the largest antenna-pair separation, while the shortest antenna-pair separation determines the "detectability" of the large scale structure. Thus, when imaging a comet with the VLA, one has to realize the following points: 1) one needs short spacings to "see" the entire object 2) one needs long spacings to achieve high spatial resolution, but 3) the long spacings should be small enough to provide a measurable flux density per beam. It turns out that the most compact VLA configuration, the D-configuration, gives the best, and so far only, results. In this configuration, the 9 antennas along each arm are spread out over approximately $600 \mathrm{~m}$. In more extended array configurations, the flux density per beam is below the detection limit, and we say the object gets "resolved" out (Schenewerk et al., 1986).

For practical reasons, in order to construct an image of an object, the responses of all the individual interferometer pairs, the visibility data, are gridded into cells having uniform intervals in projected antenna-pair separation on the sky. These spacings are measured in the North-South, called $u$, and East-West, called $v$, directions; this is commonly referred to as the $u v$ plane. This grid of data is then Fourier transformed to give an image of the brightness distribution (Bracewell, 1979). This process requires a weighting function for the $u v$ data (see Sramek, 1982; Sramek and Schwab, 1989). One typically uses either a natural or a uniform weighting function. In the first option, each cell is given a weight proportional to the number of data points inside that cell. Since for the VLA the cells near the $u v$ origin contain relatively more data points than the cells elsewhere in the $u v$ plane, the short spacing data are weighted quite heavily, which produces a rather broad beam, with large sidelobes. It gives the best signal-to-noise ratio and hence is best for detection experiments and for mapping weak sources. In the uniform weight option each cell in the $u v$ plane is given equal weight. In this case, the size of the beam is smaller, and the sidelobes are reduced. For $\mathrm{OH}$ mapping of comets the natural weight option gives the best results.

Once the image is obtained, one may need to remove the response of the antenna beam from the images (see e.g. Cornwell and Braun, 1988). If the object is weak, and the $u v$ plane well sampled (as for $\mathrm{OH}$ observations in the D-configuration), one can usually suffice with the uncleaned (commonly called "dirty") images. On the other hand, if the plane is not well sampled (as for observations in a hybrid configuration), large sidelobes exist which may distort the picture. In those cases one needs to deconvolve the image to remove the antenna beam response. The most widely used technique is CLEAN (Högbom, 1974; Clark, 1980). CLEAN deconvolves an image by an iterative procedure in which a fraction of the interferometer response to the brightest point remaining on the map is successively subtracted. This process is repeated until the entire source is removed from the map. The CLEAN-components are then convolved with the Gaussian beam which best fits the central part of the antenna pattern and restored to the map.

To get an idea of the size and brightness distribution one can image with the VLA, we modeled various objects. Figure la shows the total flux density as a function of source size, which would be measured with the VLA in the D-configuration. We assumed a Gaussian 

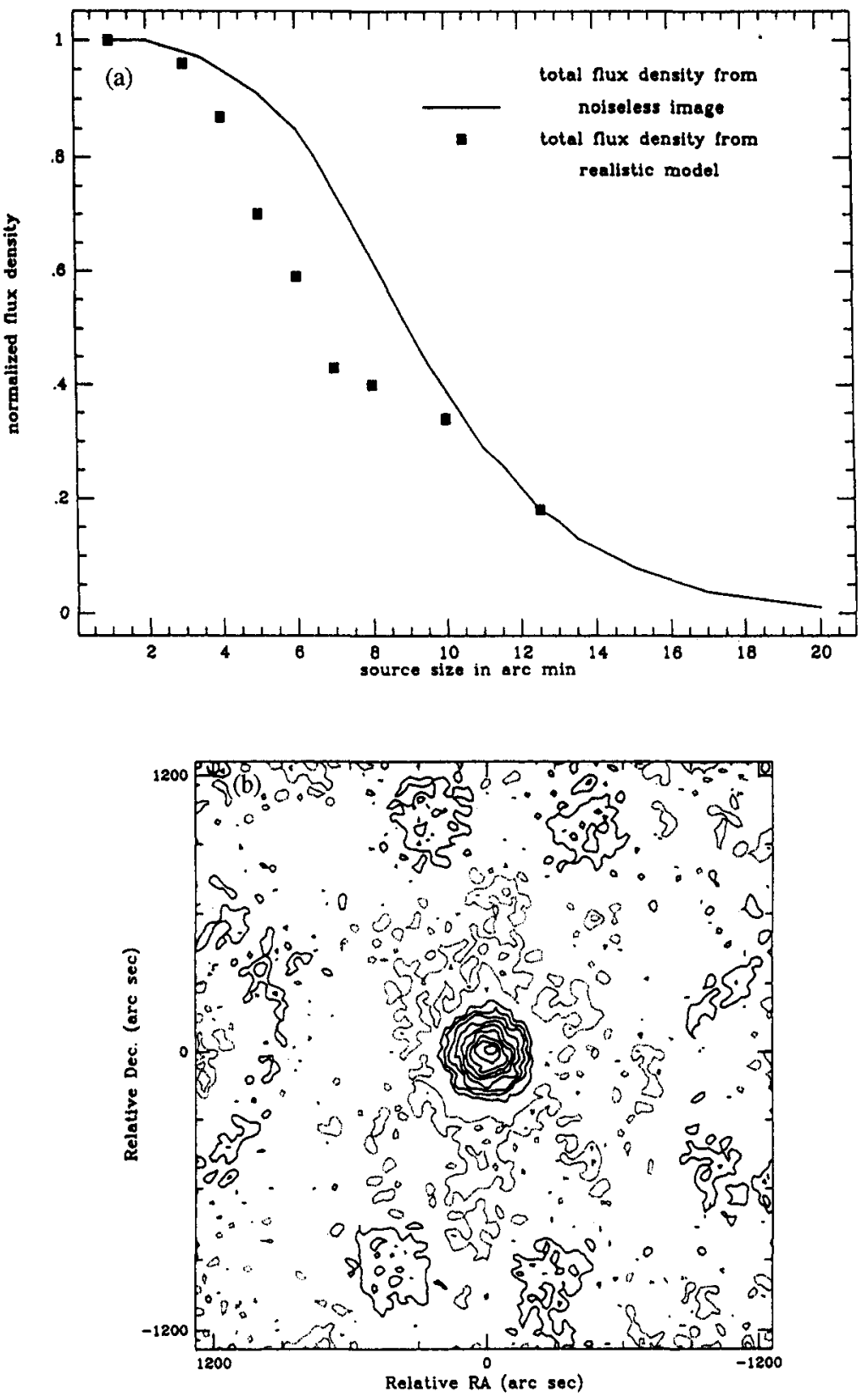

Fig. 1. (a) Flux density received by the VLA in the D-configuration from a source with a Gaussian brightness distribution. The declination of the source was taken as $20^{\circ}$, the integration time as $8 \mathrm{hrs}$, and the total flux density of the source as $1 \mathrm{Jy}$. The solid line is for a source in a noiseless medium, and the squares for an image with $\sim 3 \mathrm{mJy}$ rms noise. (b) Image of a model Gaussian source with a size of 4'. The total flux density of the model source is $1 \mathrm{Jy}$, the peak flux density is $82 \mathrm{mJy} / \mathrm{beam}$, and the contour interval is in steps of $10 \%$. 
distribution for a source at $20^{\circ}$ declination and a total flux density of $1 \mathrm{Jy}$ in a noiseless medium (solid line). The total "observing" time was $8 \mathrm{hrs,} \mathrm{to} \mathrm{closely} \mathrm{match} \mathrm{observations} \mathrm{of}$ Comet Halley taken in November, 1985 (de Pater, Palmer and Snyder, 1986). (The shape of the curves will vary somewhat with the source declination and integration time.) The graph shows that we will receive over $90 \%$ of the flux density if the source is less than $\sim 5^{\prime}$ (FWHM). Sources as large as $10-12$ ' are still "visible" to the VLA, but the total flux density received is only $\sim 20-30 \%$ of the total emission. For comparison, if the lifetime of $O H$ is $2 \times 10^{5}$ seconds, and the outflow velocity is $1 \mathrm{~km} / \mathrm{sec}$, a source with a radius of the scalelength of $\mathrm{OH}$ will subtend an angle of $9.2^{\prime}$ at $1 \mathrm{AU}$. If we include a typical noise of $3 \mathrm{mJy} / \mathrm{beam}$ in $8 \mathrm{hrs}$ for the model in Fig. 1a, we find the squares indicated on the graph. This curve shows that the source size should be limited to $3^{\prime}$ rather than $5^{\prime}$, if we want to receive over $90 \%$ of the flux density. Figure $1 \mathrm{~b}$ displays an image of a model with a $4^{\prime}$ diameter Gaussian source with an rms noise of $3 \mathrm{mJy} /$ beam. No CLEANing algorithms were used to deconvolve the picture. As shown in Fig. 1a, we receive roughly $60 \%$ of the flux for this particular case. The source itself is clearly visible at the center of the map. It is surrounded by negative contours, indicative of the bowl-like feature expected when one observes a relatively large source with an interferometer. This feature will be somewhat suppressed when the image is CLEANed; in order to remove it entirely, short spacing (single dish) data need to be included. Small, discrete features can be imaged well with the VLA, as long as the emissions are within the primary beam of the telescope ( $\sim 30^{\prime}$ for $\mathrm{OH}$ observations).

The actual fraction of flux detected by the VLA depends on the shape of the source, and a Gaussian is the worst case. For example, for a Gaussian source with FWHM of $10^{\prime}$, one measures about $55 \%$ of the visibility amplitude with the VLA; for a disk with a diameter of $10^{\prime}$, one measures $\sim 80 \%$. Finally, we note that while we have discussed this spatial filtering property of an array as a limitation, the converse view should be made clear as well: If the fraction of the flux density from an extended object measured with the VLA is larger than expected for a smooth source, the object must have small-scale structure.

The effect of the telescope beam on a comet's line profile and total flux density is shown schematically in Figs. $2 \mathrm{a}$ and $2 \mathrm{~b}$. Since we want to show only the effect of different telescope beams on a comet's line profile and its total flux density, we assumed the Haser model for its outgassing. We adopted a geocentric distance of $1.44 \mathrm{AU}$, and an outflow velocity of $1.0 \mathrm{~km} / \mathrm{sec}$. The lifetimes for the parent and daughter molecules were taken, respectively, as $8 \times 10^{4} \mathrm{sec}$ and $2 \times 10^{5} \mathrm{sec}$ at $1 \mathrm{AU}$. The profiles in Fig. $2 \mathrm{a}$ show the effect of spatial integration: a $1^{\prime}$ beam shows two peaks in emission at $\pm 0.7 \mathrm{~km} / \mathrm{sec}$. For larger beam sizes the peaks move inward, and for a beam size of $30^{\prime}$ the top of the profile is flat. Obviously, the flux density increases with beam size as well. Also, we indicate the profile for a box integration of $6.5^{\prime} \times 9.5^{\prime}$ to imitate a profile for VLA data, if the previously discussed missing short spacing problem can be ignored (see Fig. 1). Figure $2 \mathrm{~b}$ shows the peak flux densities of these curves as a function of the half power width of a Gaussian telescope beam. (In this plot, the elliptical beam of the Nancay telescope corresponds to about $5.8^{\prime}$ for this source model.)

From Figs. $2 \mathrm{a}$ and $2 \mathrm{~b}$ it should be clear that the true total flux density can only be measured with small single dishes. With larger single dishes, one measures a smaller fraction of the flux. For the observations of Comet Halley, Galt's (1987) beam was about $30^{\prime}$, Schloerb, Claussen and Tacconi-Garman's (1987) about 18', and the beam of the Nancay telescope is roughly $3.5^{\prime} \times 19^{\prime}$ (Gerard et al., 1987; 1989). The primary beam of the VLA 

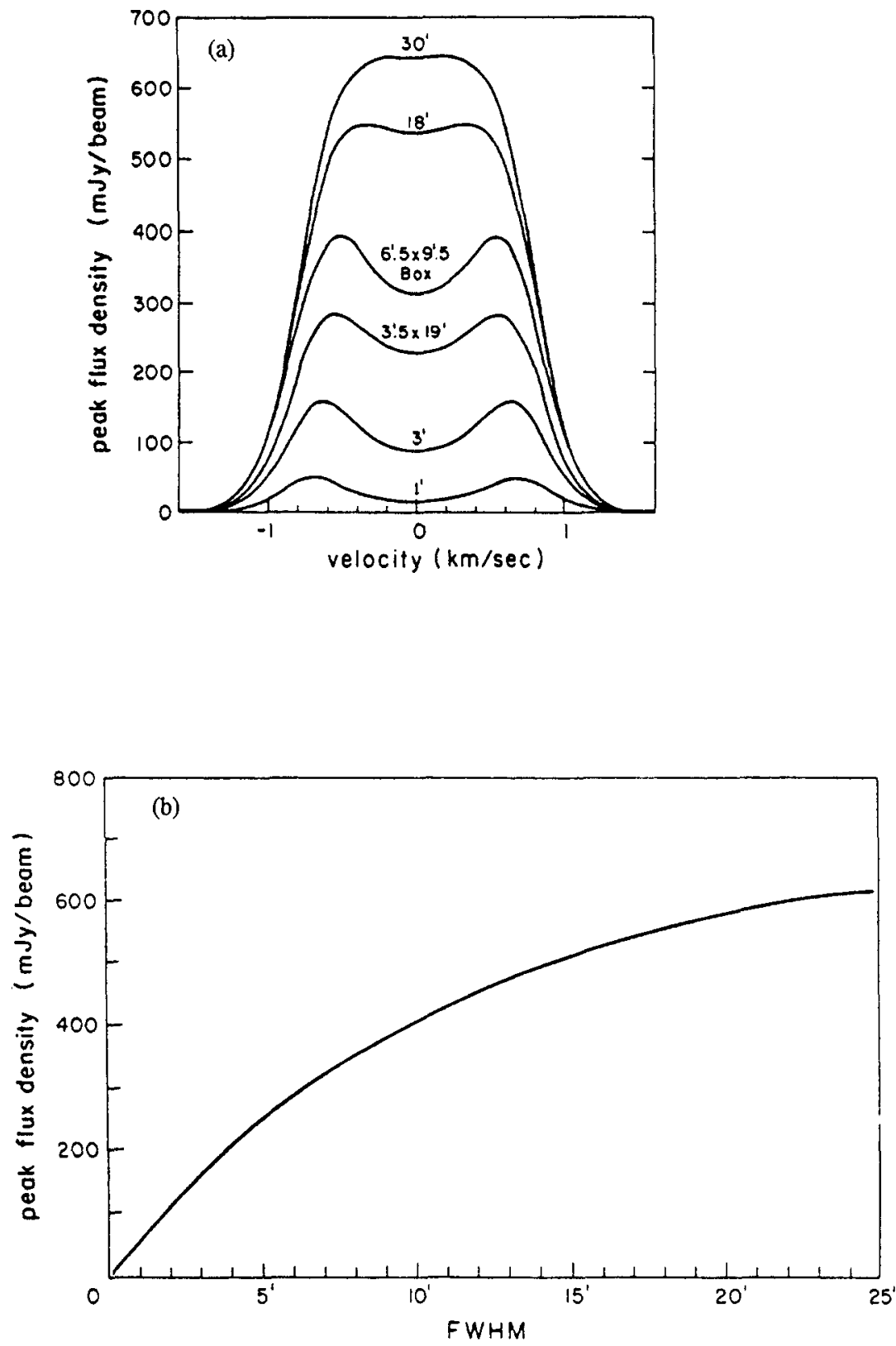

Fig. 2. (a) The effect of spatial integration on the $O H$ spectral line profiles. (b) The peak flux densities of the profiles in (a) as a function of telescope beam width. The curves are for a comet that is at $1.44 \mathrm{AU}$ and that is outgassing according to a Haser model, with an outflow velocity of $1 \mathrm{~km} / \mathrm{sec}$, and lifetimes for the parent and $\mathrm{OH}$ molecules of $8 \times 10^{4} \mathrm{sec}$ and $2 \times 10^{5} \mathrm{sec}$, respectively. 
is roughly $30^{\prime}$. Although the flux per synthesized VLA beam $\left(\sim 1^{\prime}-2^{\prime}\right)$ will be smaller than that observed with the Nancay telescope, the flux integrated over a VLA image might be larger than that observed at Nancay, if the source contains small-scale structure that is outside the Nancay beam.

\section{VLA Observations}

Since August 1982, we have observed a number of comets; a complete list of VLA attempts since that time is shown in Table 1 . The name of the comet is listed in column 1 , the observing date in column 2 , the VLA configuration and observing wavelength in columns 3 and 4 respectively. Column 5 shows the types of experiments which were attempted. Successful observations are indicated by $a+$ in front of the experiment. As shown, successful observing runs have been rare. The last column gives the reference if the data were published, or the state of reduction and data analysis. In the latter case, we indicated the likelihood that we will be able to publish meaningful results. Terms like "hopeless" and "grim" speak for themselves.

\subsection{CONTINUUM OBSERVATIONS}

Continuum observations of comets have been attempted regularly over the past 20 years. Several detections at the 3-4 $\sigma$ level were reported (Hobbs et al., 1975; 1977) at a wavelength of $3.7 \mathrm{~cm}$. Observations of weak sources at $\mathrm{cm}$ wavelengths with single element radio telescopes are very difficult, since confusion plays an important role. Continuum measurements were made of comets Iras-Araki-Alcock and Halley at $1.3 \mathrm{~cm}$ and $1.3-3.5 \mathrm{~mm}$ (Altenhoff et al., 1983; 1986). The close passage of Comet Iras-Araki-Alcock and consequent rapid proper motion contributed to the success of the $1.3 \mathrm{~cm}$ observations. More reliable measurements can be obtained when using an interferometer like the VLA. This has been done a few times, as indicated in Table 1. All experiments were negative in that no emission was observed. It is interesting to note here that Altenhoff et al. (1983) detected $1.3 \mathrm{~cm}$ emission from Comet Iras-Araki-Alcock, while VLA observations at 2 and $6 \mathrm{~cm}$ did not show a detection. The $3 \sigma$ upper limit at $2 \mathrm{~cm}$ was 4 times less $(750 \mu \mathrm{Jy})$ than that expected from a blackbody with a flux density of $9 \mathrm{mJy}$ at $1.3 \mathrm{~cm}$ as observed. De Pater et al. (1985) show that it is conceivable that one probes rather different regions in a cometary nucleus at 1.3 and $2 \mathrm{~cm}$. Typically, the $1.3 \mathrm{~cm}$ observations pertain to a depth closer to the surface than the $2 \mathrm{~cm}$ data. Hence, the $1.3 \mathrm{~cm}$ detection together with the $2 \mathrm{~cm}$ non-detection implies a steep temperature gradient in the nucleus. A similar conclusion can be reached when comparing the detections of Comet Halley at $\mathrm{mm}$ wavelengths by Altenhoff et al. (1986) with the VLA non-detection at $2 \mathrm{~cm}$ (Hoban and Baum, 1987). Other possibilities to explain the continuum emission are reviewed by Crovisier and Schloerb (1990).

\subsection{OCCULTATION EXPERIMENTS}

In a refraction experiment one monitors a background radio source to look for refraction or scintillation as the comet occults the source. Ananthakrishnan, Manoharan and Venugopal (1987) conclude that none of the attempted scintillation experiments on comets showed an unambiguous increase in scintillation as the comet occults the radio source. In a refraction 
Table 1. Experiments carried out with VLA over past years ${ }^{\mathbf{a}}$

\begin{tabular}{|c|c|c|c|c|c|c|}
\hline Comet & & Date (IAT) & $\begin{array}{l}\text { VLA } \\
\text { Conf. }\end{array}$ & $\begin{array}{c}\text { Wave- } \\
\text { length, } \\
\mathrm{cm}\end{array}$ & Experiment $b$ & $\begin{array}{c}\text { Reference } \\
\text { or State } \\
\text { of Reductions }\end{array}$ \\
\hline Austin & 1982 & Aug/Sep & $\begin{array}{l}\mathrm{B} \\
\mathrm{B} \\
\mathrm{B} \\
\mathrm{B}\end{array}$ & $\begin{array}{c}6 \\
1.3-6 \\
20 \\
18 \\
\end{array}$ & $\begin{array}{l}\text { Continuum } \\
\text { Continuum } \\
\text { Refraction } \\
\text { OH Imaging }\end{array}$ & $\begin{array}{l}\text { Snyder et al., } 1983 \\
\text { de Pater and Ip, } 1984 \\
\text { de Pater and Ip, } 1984 \\
\text { Schenewerk et al., } 1986 \\
\end{array}$ \\
\hline Crommelin & 1984 & $\begin{array}{l}\text { Mar } 09 \\
\operatorname{Jan} 25 \\
\end{array}$ & $\begin{array}{c}\mathrm{B} / \mathrm{C} \\
\mathrm{B} \\
\end{array}$ & $\begin{array}{c}2 \\
18 \\
\end{array}$ & $\begin{array}{l}\text { Continuum } \\
\text { OH Imaging }\end{array}$ & $\begin{array}{l}\text { Schenewerk et al., } 1986 \\
\text { Schenewerk et al., } 1986\end{array}$ \\
\hline $\begin{array}{l}\text { IRAS-Araki- } \\
\text { Alcock }\end{array}$ & 1983 & $\begin{array}{l}\text { May } 08 \\
\text { May } 12 \\
\end{array}$ & $\begin{array}{l}\mathrm{C} \\
\mathrm{C}\end{array}$ & $\begin{array}{l}6 \\
2 \\
\end{array}$ & $\begin{array}{l}\text { Continuum } \\
\text { Continuum } \\
\end{array}$ & de Pater et al., 1985 \\
\hline $\begin{array}{c}\text { Giacobini- } \\
\text { Zinner }\end{array}$ & 1985 & $\begin{array}{l}\text { Sep } 07 \\
\text { Sep } 15 \\
\end{array}$ & $\begin{array}{l}\mathrm{C} \\
\mathrm{C}\end{array}$ & $\begin{array}{l}18 \\
20 \\
\end{array}$ & $\begin{array}{c}\text { OH Imaging } \\
\text { Refraction }\end{array}$ & $\begin{array}{l}\text { (In progress-grim) } \\
\text { (Hopeless??) }\end{array}$ \\
\hline Halley & $\begin{array}{l}1985 \\
1986\end{array}$ & $\begin{array}{l}\text { Nov } 13,16 \\
\text { Nov } 17 \\
\text { Dec } 04,05 \\
\text { Jan } 04,06 \\
\text { Jan } 12,26,31 \\
\text { Apr } 02,19 \\
\text { Apr } 24 \\
\end{array}$ & $\begin{array}{l}\mathrm{D} \\
\mathrm{D} \\
\mathrm{D} \\
\mathrm{D} \\
\mathrm{D} \\
\mathrm{A} \\
\mathrm{A}\end{array}$ & $\begin{array}{c}18 \\
2 \\
18 \\
18 \\
\\
6 \\
20 \\
18 \\
\end{array}$ & $\begin{array}{l}\text { + OH Imaging } \\
\text { Continuum } \\
\text { OH Occultation } \\
\text { + OH Imaging } \\
\\
\text { + Formaldehyde } \\
\text { Refraction } \\
\text { OH Occultation } \\
\end{array}$ & $\begin{array}{l}\text { de Pater et al., 1986a } \\
\text { Hoban and Baum, } 1987 \\
\text { (In progress-grim) } \\
\text { de Pater et al., 1986b; } \\
\text { this paper } \\
\text { Snyder et al., 1989a } \\
\text { (Hopeless??) } \\
\text { (Hopeless??) }\end{array}$ \\
\hline Wilson & 1987 & $\begin{array}{l}\text { Feb 06, } 07 \\
\text { Feb 28, Mar } 03\end{array}$ & $\begin{array}{c}C D \\
D\end{array}$ & $\begin{array}{l}18 \\
18 \\
\end{array}$ & $\begin{array}{l}+\mathrm{OH} \text { Imaging } \\
+\mathrm{OH} \text { Imaging }\end{array}$ & $\begin{array}{l}\text { Palmer et al., } 1989 \\
\text { Palmer et al., } 1989\end{array}$ \\
\hline Liller & 1988 & $\operatorname{Mar} 22,23$ & $\mathrm{C}$ & 18 & $\mathrm{OH}$ Imaging & (In progress) \\
\hline Machholz & 1988 & $\begin{array}{l}\text { Sep } 15 \\
\text { Sep } 16,20\end{array}$ & $\begin{array}{l}\mathrm{D} \\
\mathrm{D}\end{array}$ & $\begin{array}{c}6 \\
18\end{array}$ & $\begin{array}{c}\text { + Formaldehyde } \\
\text { OH Imaging }\end{array}$ & $\begin{array}{l}\text { Snyder et al., 1989b } \\
\text { (In progress-grim) }\end{array}$ \\
\hline
\end{tabular}

a Positive detections are indicated by a "+" before the experiment type.

$b$ The experiments were as follows:

Continuum: Searching for the microwave continuum emission.

Refraction: Monitoring of a background source to look for refraction or scintillation as the comet occults the source.

OH Imaging: Imaging of the comet in the $18-\mathrm{cm} \mathrm{OH}$ line.

OH Occultation: Monitoring of the background radio source over a spectral band expected to contain the $\mathrm{OH}$ in the comet to look for absorption or emission in the $\mathrm{OH}$ lines modulated by the background source.

Formaldehyde: Searching for the 6-cm formaldehyde line from the comet. 
experiment, the apparent position of the source on the sky is monitored. A change in the source's position can be due to a gradient in the cometary plasma, and can be used to place limits to the electron number density (Wright and Nelson, 1979). However, just like in a scintillation experiment, it is very difficult to distinguish real cometary effects from ionospheric fluctuations. Although several refraction experiments have been done with the VLA (Table 1), no successful unambiguous observations have yet been reported.

\subsection{OH OBSERVATIONS}

Although $\mathrm{OH}$ has been monitored regularly with single element radio telescopes (Crovisier and Schloerb, 1990), high resolution images can only be obtained with help of interferometers. As shown in Table 1, several comets have been imaged over the past few years; the results will be discussed in Sections 4 and 5 . All VLA observations were taken at the ${ }^{2} \Pi_{3 / 2}, J=3 / 2, F=2-2$ transition at $1667.3590 \mathrm{MHz}$, with a resolution of $3.052 \mathrm{KHz}$ $(0.55 \mathrm{~km} / \mathrm{sec})$.

In addition to imaging, occultation experiments of a background radio source in the $\mathrm{OH}$ line have been attempted. Although a detection was made, interpretation of the signal is complicated, and therefore not yet finished.

\subsection{SEARCH FOR OTHER SPECTRAL LINES}

We have shown that interferometers are very sensitive for the detection of weak cometary lines. One constructs a data cube (Rots, 1989; Snyder, Palmer and de Pater, 1989a): a series of images of the comet in all velocity channels, where the velocity can be viewed as the third dimension. Spectral line profiles at the position of the comet can be obtained by making a cut through the data cube. Application of Gerard's (1987) SYMCOMET routine can be applied to the spectrum to increase the signal-to-noise level in the profile. In this routine, a search for a symmetrical spectral line is conducted by folding the spectrum about an axis which is sequentially stepped through the velocity channels. With this method successful observations of the $J_{K_{-1} K_{1}}=1_{11} 1_{10}$ transition of formaldehyde were obtained for the comets Halley and Machholz (Snyder, Palmer, and de Pater, 1989a,b).

Snyder, Palmer and de Pater (1989a) made the first unambiguous detection of formaldehyde. They show that the formaldehyde must come from an extended source in the coma, rather than only the nucleus itself. The derived scalelength is in good agreement with the in situ measurements of $\mathrm{CO}$ (Eberhardt et al., 1987), and was later confirmed by Krankowsky (1990) from $\mathrm{H}_{2} \mathrm{CO}$ measurements made with the Giotto neutral gas mass spectrometer. In addition to the $\mathrm{H}_{2} \mathrm{CO}$ detection of Comet Halley, Snyder, Palmer and de Pater (1989b) detected formaldehyde emission from Comet Machholz. A comparison of the two detections is shown in Fig. 3. The lines are slightly blueshifted for both comets $(-0.76 \pm 0.40 \mathrm{~km} / \mathrm{sec})$. The $\mathrm{H}_{2} \mathrm{CO}$ production rate from Comet Machholz is an order of magnitude larger than that for Comet Halley. The fractional production rate for the latter comet is $\sim 1.5 \%$; the fractional production rate from Comet Machholz could not be determined since the $\mathrm{H}_{2} \mathrm{O}$ production rate was not measured at the time. The $\mathrm{H}_{2} \mathrm{CO}$ line in Comet Machholz is $\sim 2.5$ times wider than that of the Comet Halley line, which may indicate that the comet had started to come apart during the VLA observations. 


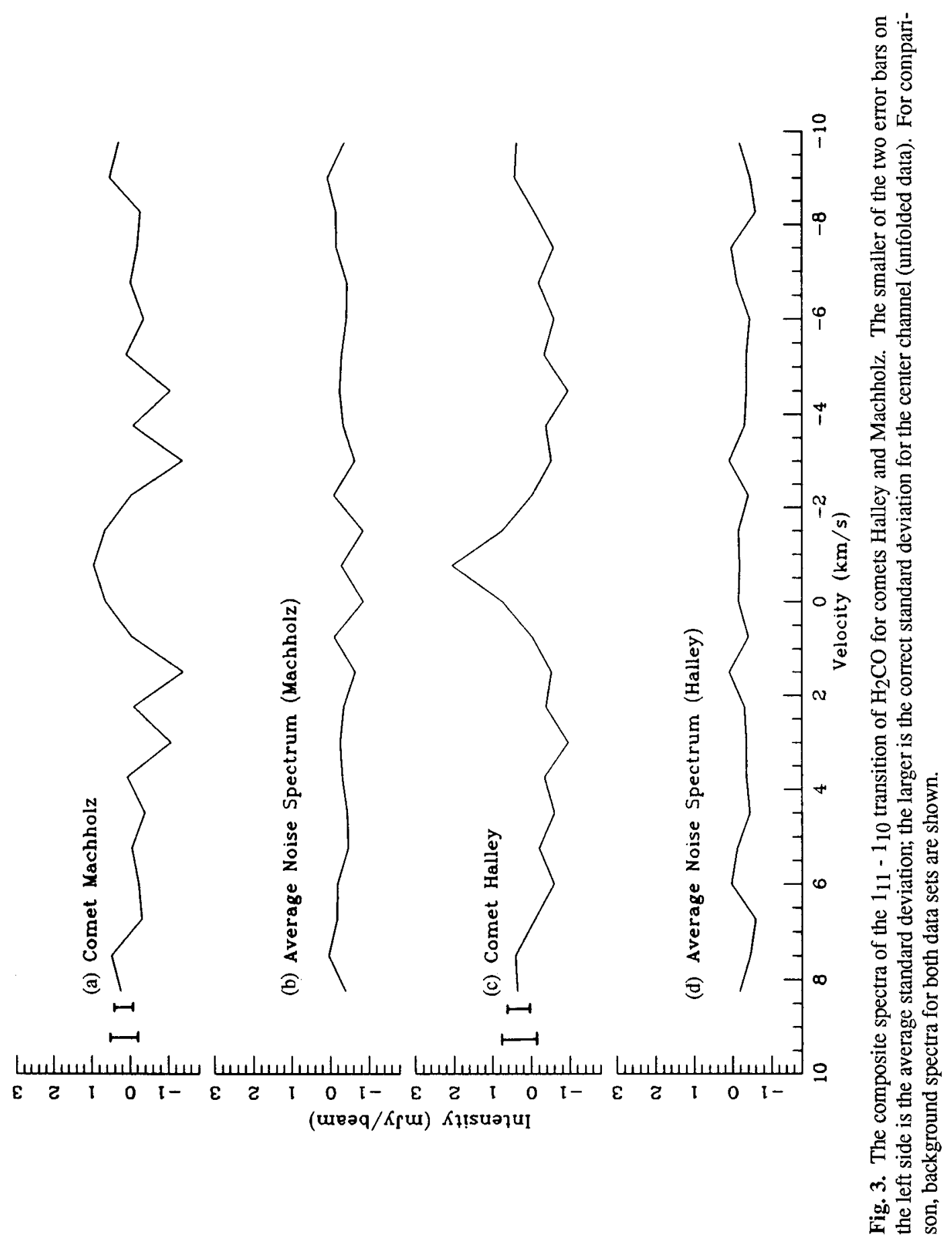




\section{Results of $\mathrm{OH}$ imaging with the VLA}

Imaging the $\mathrm{OH}$ coma has been rather successful over the past few years. After an initial non-detection of comets Austin and Crommelin (Schenewerk et al.,1986), several images have been obtained of comets Halley and Wilson. These will be discussed in detail in this Section.

\subsection{TOTAL FLUX DENSITY}

The viewing geometries of the comets Halley and Wilson are shown in Fig. 4. Table 2 contains data on the flux density at the velocity of peak emission of the line for both Comet Halley and Comet Wilson, measured by the VLA and several single dishes. Because of the variability of the $\mathrm{OH}$ signal, we have only used data taken on the same days as the VLA observations. For each telescope, the velocity of the peak flux, and an estimate of the half power line width is given. The errors quoted for the VLA are our best estimates of the errors. The errors quoted for the single dishes are statistical errors of Gaussian fits; in general we have used the fits allowing the maximum number of free parameters when the authors published several sets of numbers. One can see that we received roughly $30 \%$ of the flux density from Comet Halley in November compared to that measured with the Green Bank telescope, and $\sim 70 \%$ in January. Note that the Green Bank telescope received only a fraction of the flux density as measured by the Penticton telescope. Hence, the emission received by the VLA is a very small fraction $(\sim 20 \%)$ of the total emission. The difference in flux density received in November and January is partly due to a change in apparent size of the comet: in November, the comet was at a geocentric distance of $0.75 \mathrm{AU}$, while it was at $1.25 \mathrm{AU}$ in early January. In addition, the lifetime of the molecules scales with the heliocentric distance squared, so the absolute size of the $\mathrm{OH}$ coma must have been larger in November than in January.

Comet Wilson was observed at a larger geocentric distance $(2.6-2.4 \mathrm{AU})$ and similar heliocentric distance than was Comet Halley, so we received most of the flux density from this comet. If the comet's brightness distribution were Gaussian in appearance, Fig. 1a shows that the source must be confined to a region of $1-2 \times 10^{5} \mathrm{~km}$. If the source shows discrete structures, it may extend well beyond this limit. The images of the comets (see below) show that Comet Halley is more compact, with a size a few times $10^{5} \mathrm{~km}$, while Comet Wilson shows blobs of emission at much larger distances $\left(\sim 10^{6} \mathrm{~km}\right)$ from the comet. Hence, the latter source must consist primarily of discrete emission blobs which can show up at relatively large distances from the center, while Comet Halley may be more Gaussian shaped with irregularities superimposed.

\subsection{SPATIAL BRIGHTNESS DISTRIBUTION}

4.2.1 Comet Halley. Figures 5 and 6 show images of Comet Halley: Figure 5 shows the image obtained at the velocity of maximum emission $(v=0.0 \mathrm{~km} / \mathrm{sec}$ in the rest frame of the comet) after combining the data from November 13 and 16. Figure $5 \mathrm{a}$ shows a lowresolution (120") image, and Fig. 5b a high-resolution $\left(60^{\prime \prime}\right)$ one. These images have not been processed by CLEANing or other deconvolution techniques. The cross in the center of the images indicates the ephemeridal position of the comet. The coordinates are offsets from this position (in arcsec) in right ascension and declination. The projected direction to 

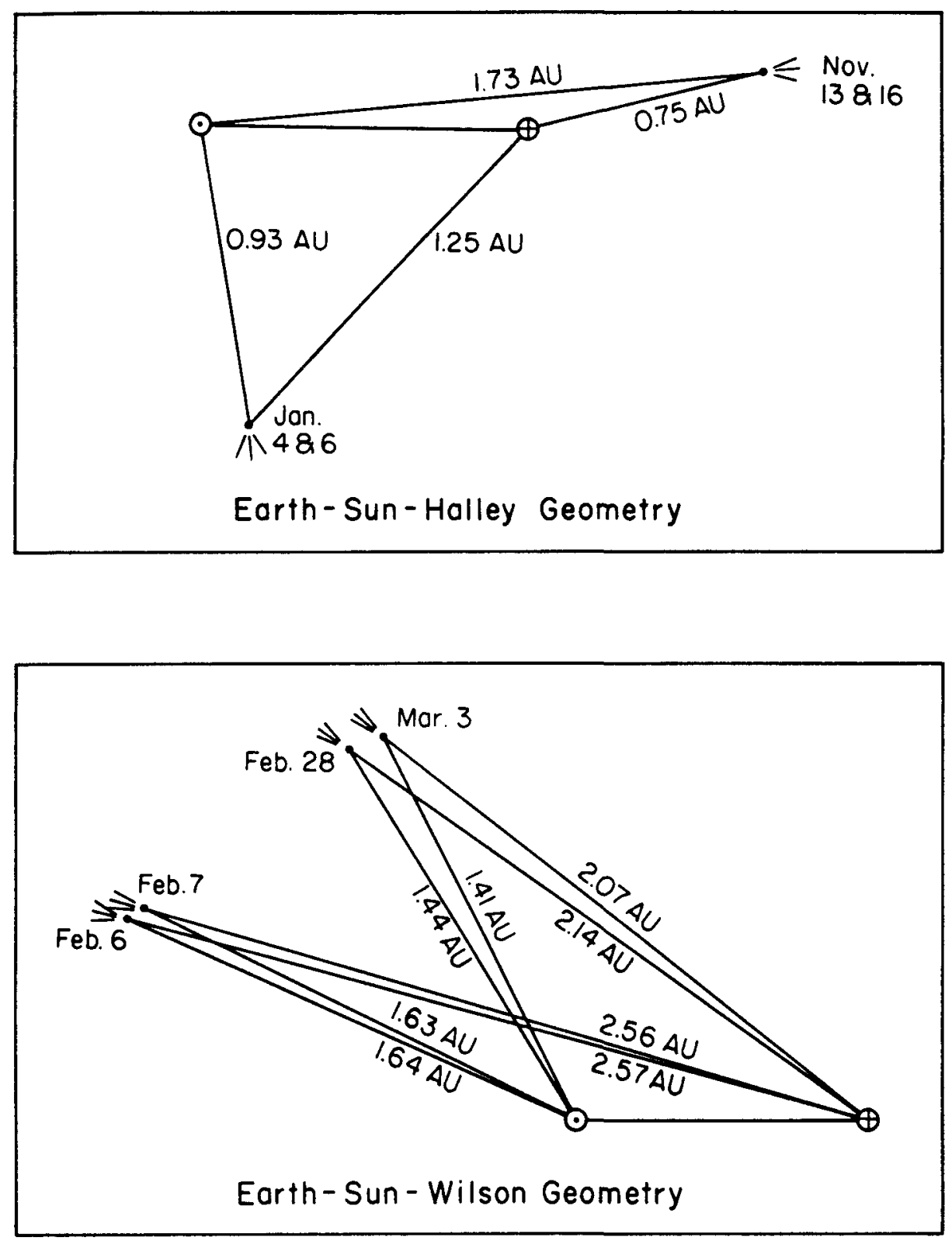

Fig. 4. The Earth-Sun — comet geometries during the VLA observations of comets Halley and Wilson. 


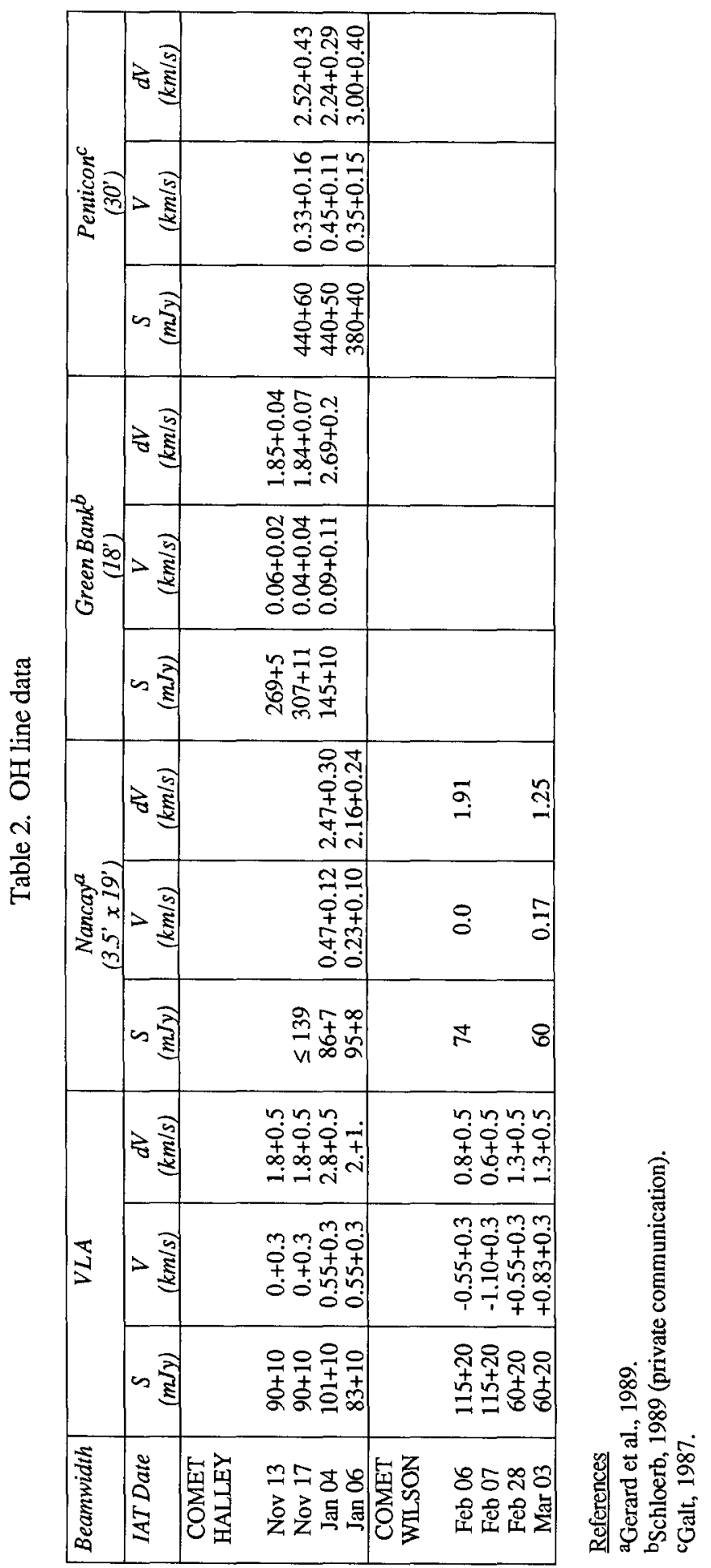



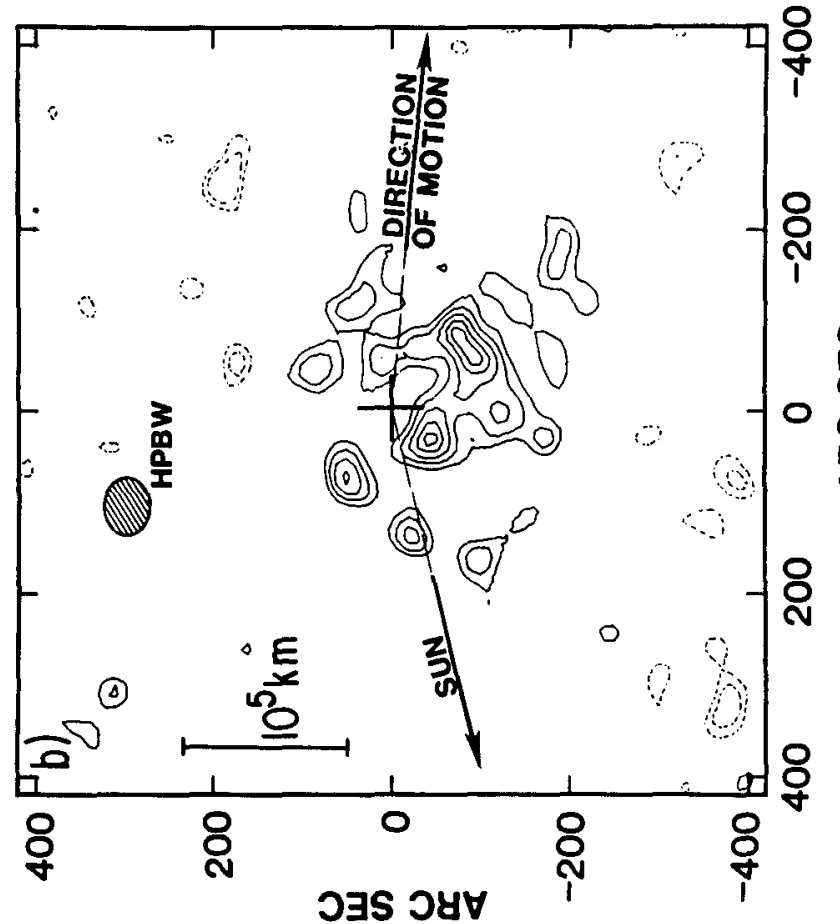

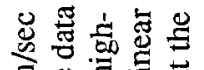

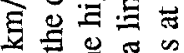

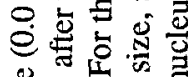

ᄋ 要

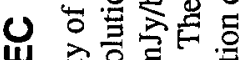

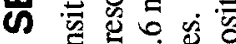

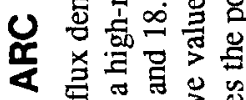

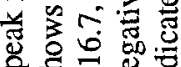

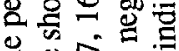

五它焉昘

运

光

8

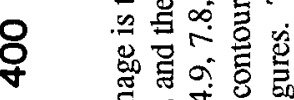

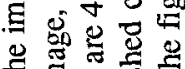

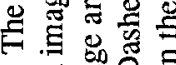

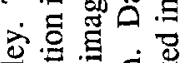

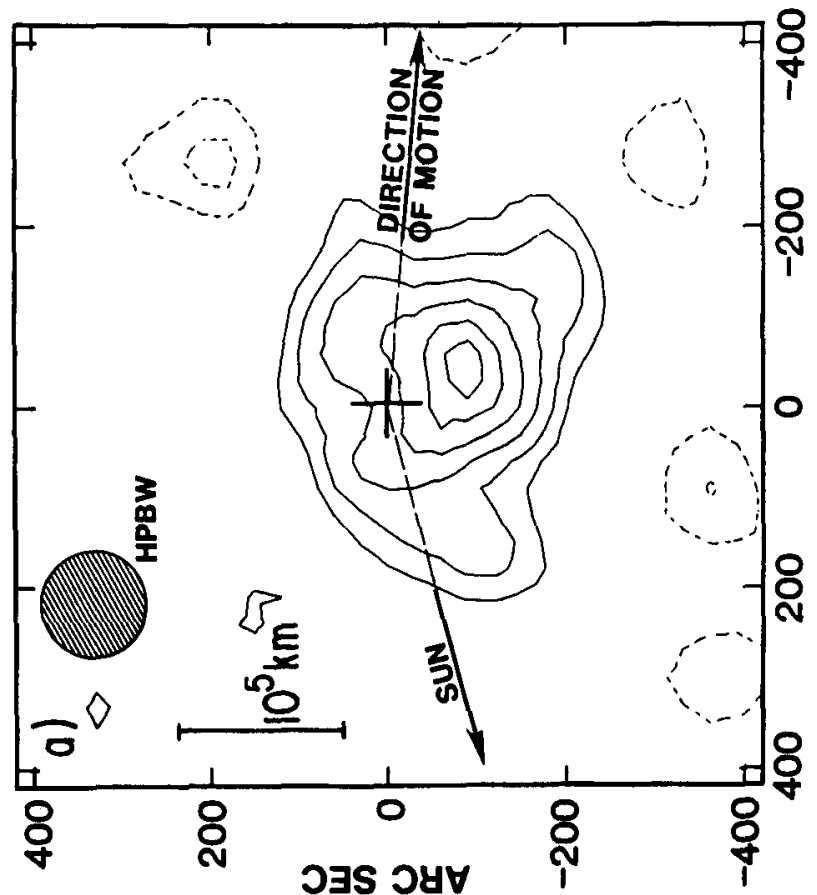

丞 귱융

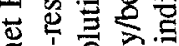

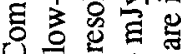

范芯苍泀

응요월

m 훙

m 믄 ํㅜㅇㅇ

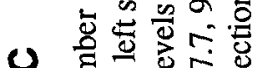

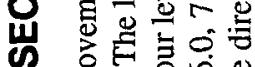

( ) 후월

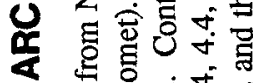

芯过

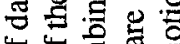

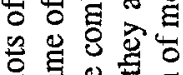
음 톤

苛范骂

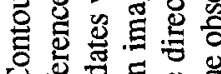

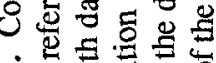

in

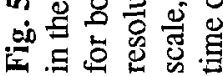



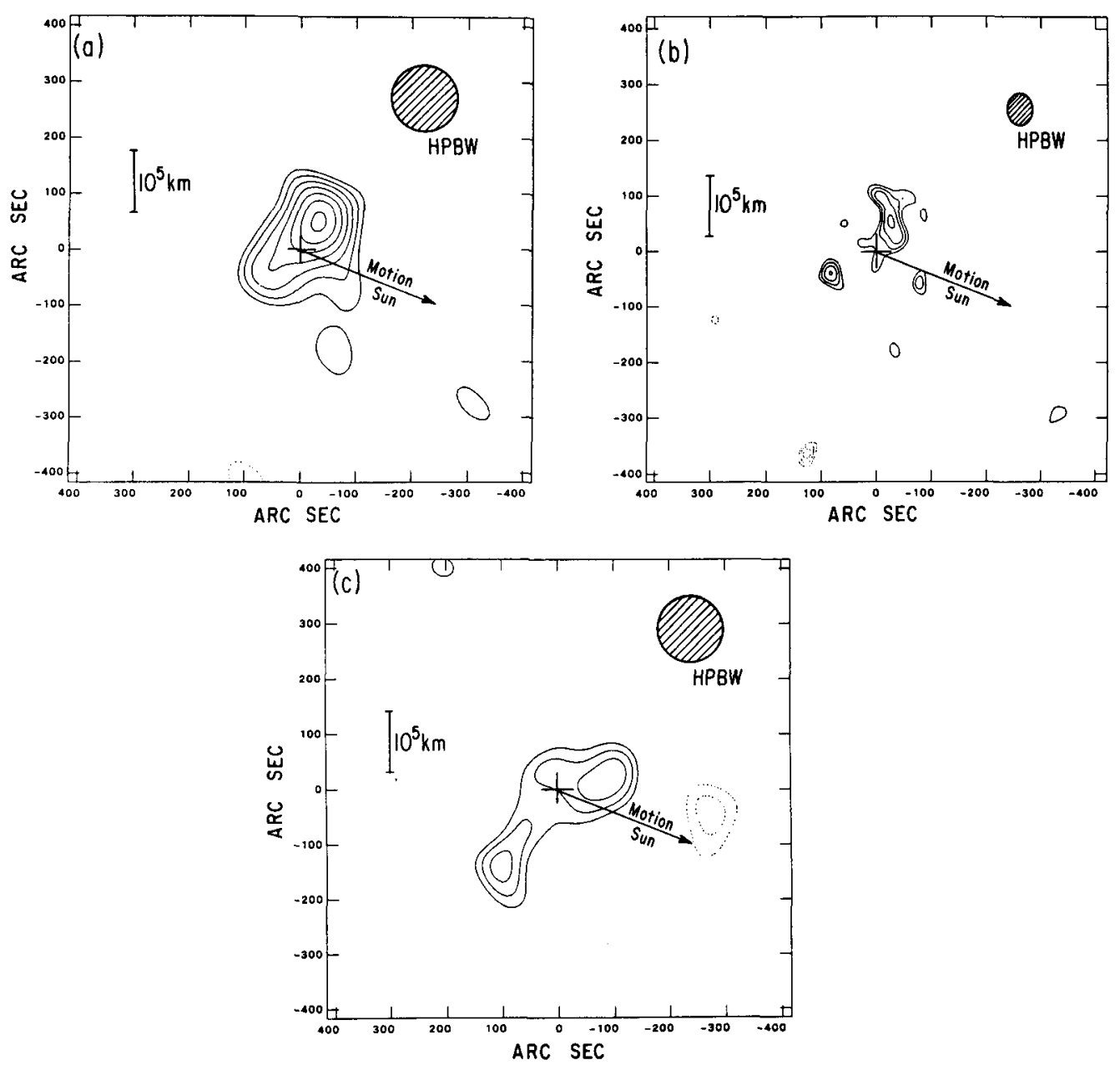

Fig. 6. Contour plots of the data from January 4 to 6 of Comet Halley at the peak flux density of the line $(+0.55 \mathrm{~km} / \mathrm{sec}$ in the reference frame of the comet). (a) A low-resolution image from January 4. Contour values are 1.5 to $3.0 \mathrm{mJy} / \mathrm{beam}$, in steps of $0.25 \mathrm{mJy} / \mathrm{beam}$. (b) A highresolution image from January 4. Contour values are 1.05 to $2.10 \mathrm{mJy} / \mathrm{beam}$, in steps of $0.175 \mathrm{mJy} / \mathrm{beam}$. (c) A low-resolution image from January 6 . To increase the signal-to-noise, the image in (c) is an average of three channels centered at velocities $0.0,+0.55$, and $+1.10 \mathrm{~km} / \mathrm{sec}$. Contour values are 0.95 to $2.06 \mathrm{mJy} / \mathrm{beam}$, in steps of $0.16 \mathrm{mJy} / \mathrm{beam}$. Negative contours on all three images are indicated by dashed lines. The beam size, linear scale, position of the nucleus, direction of motion, and direction to the Sun are indicated in the figure. 
the sun and of the comet's motion are indicated by the arrows. The half power beam width and a scale in kilometers at the distance of the comet are indicated as well. Figures 6a and $6 \mathrm{~b}$ show similar images obtained on January 4 . Since the flux density peaked at a velocity of $+0.55 \mathrm{~km} / \mathrm{sec}$, the image displayed is for $+0.55 \mathrm{~km} / \mathrm{sec}$ rather than $0.0 \mathrm{~km} / \mathrm{sec}$. On Jamuary 6 the data are noisier, and we display only a low-resolution image (Fig. 6c). To increase the signal-to-noise, we have combined the data from channels corresponding to a velocity of 0.0 , +0.55 , and $+1.10 \mathrm{~km} / \mathrm{sec}$. (A similar combination for the January 4 data hardly changes the picture displayed in Fig. 6a.)

The low-resolution images show that the peak intensity is offset from the nucleus: to the southeast in November and northeast on January 4. In November, the entire region is surrounded by negative contours, which suggests that the source size is relatively large. This fact was less pronounced in January, when the apparent coma size was smaller. Note that this phenomenon agrees with the increased fraction of the single dish flux density received in January.

The high-resolution images from November and from January 4 show that the distribution of the $\mathrm{OH}$ emission is not even approximately smooth. Whether the "clumps" in the image are discrete features or peaks on a broad emission region cannot be inferred from the present images. The positions of the clumps did not change measurably between November 13 and $16\left(<30^{\prime \prime}\right.$, implying a motion of $<60 \mathrm{~m} / \mathrm{sec}$ ). We therefore could combine the data from November 13 and 16 into one image, which improved the signal-to-noise by a factor of $\sqrt{2}$, as expected for a fixed pattern of clumps with Gaussian noise. It is remarkable that the position of these peaks hardly changed, while the total flux density showed large (typically $30 \%$ ) daily fluctuations (Galt, 1987) during the period of the November observations. Note that the central part of the image appears to be empty. For physical reasons, it is unlikely that there is no $\mathrm{OH}$ in the center of the image. Recall that we are seeing only a small fraction from the total flux density $(\sim 20 \%)$. One should think of the observed image as fine details superimposed on a smooth distribution containing the other $80 \%$ of the flux (which is invisible to the VLA for the reasons discussed in Section 2.2).

Since we were unable to obtain good high-resolution images on January 6 , we cannot compare the positions of the clumps on January 4 with those on January 6 to investigate the differential motion of these clumps in this time period. The low resolution image, however, implies that the structure did change between January 4 and January 6 . From the single dish data from Galt (1987), it appears that our observations were in the middle of an $\sim 10$ day period during which the total flux density varied by a factor of $\sim 3$. If this were due to an outburst of the comet, such variations in structure should not be unexpected.

4.2.2 Comet Wilson. Figures 7 and 8 show images of Comet Wilson. Since this comet was 2-3 times further away than Comet Halley during our November observations, we could not obtain as high a resolution at the comet as in our earlier observations. The images in Fig. 7 were cleaned, while those in Fig. 8 were not (see Palmer, de Pater and Snyder, 1989). The upper panel in Fig. 7 shows images of the velocity channels with maximum emission on February 6 and February 7. These are indicated by solid contours; contours for adjacent channels are superimposed with dotted and dashed lines (see the figure caption). The peak of the emission profile shifted by one channel, from $\mathrm{v}=-0.55 \mathrm{~km} / \mathrm{sec}$ on February 6 , to $\mathrm{v}$ $=-1.10 \mathrm{~km} / \mathrm{sec}$ on February 7 , and the spatial distribution of the emission is very different between the two days. In particular, the structure changes on a scale too large to be due 

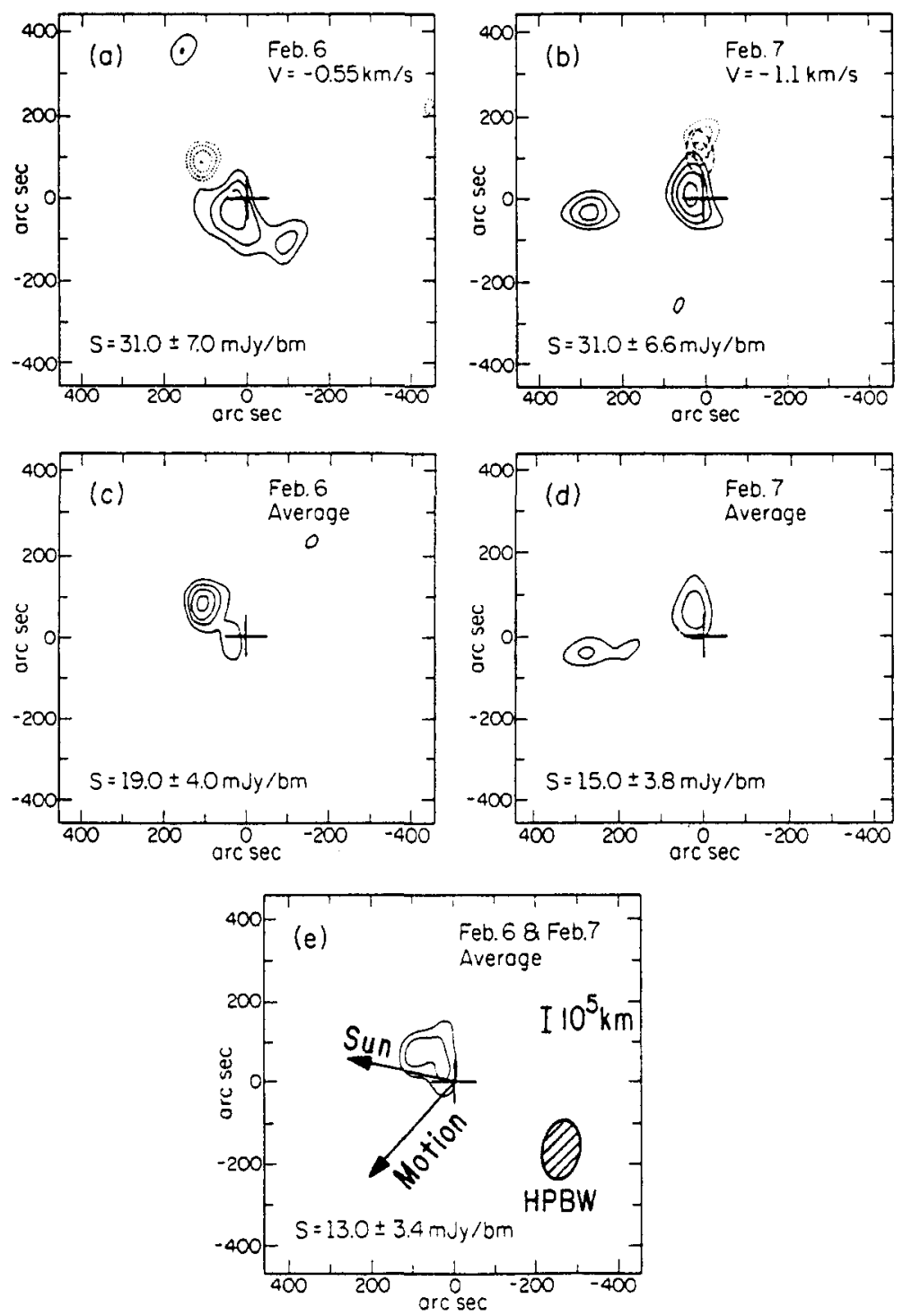

Fig. 7. Contour plots of Comet Wilson data for February 6 and 7. The contours are plotted in steps of $0.5 \sigma$, beginning with $3 \sigma$. The peak flux and sigma are given at the bottom of each panel. Panels (a) and (b) show superimposed plots for individual channels. The solid contours represent data for the velocity indicated in the panel. The dotted contours represent the channel one velocity step $(0.55 \mathrm{~km} / \mathrm{sec})$ more negative; the alternating dashes and dots represent two steps more positive. The few negative contours that showed up at the 3- $\sigma$ level had to be suppressed for clarity. Panels (c) and (d) show the average of the data for $v=0, v=-0.55$, and $v=-1.10 \mathrm{~km} / \mathrm{sec}$. Panel (e) is the average of (c) and (d). The coordinates are relative to the predicted position of the nucleus. As in Figs. 5 and 6, this position is indicated by a cross; the beam size, an angular scale, the direction of motion, and the direction to the Sun are indicated as well. 

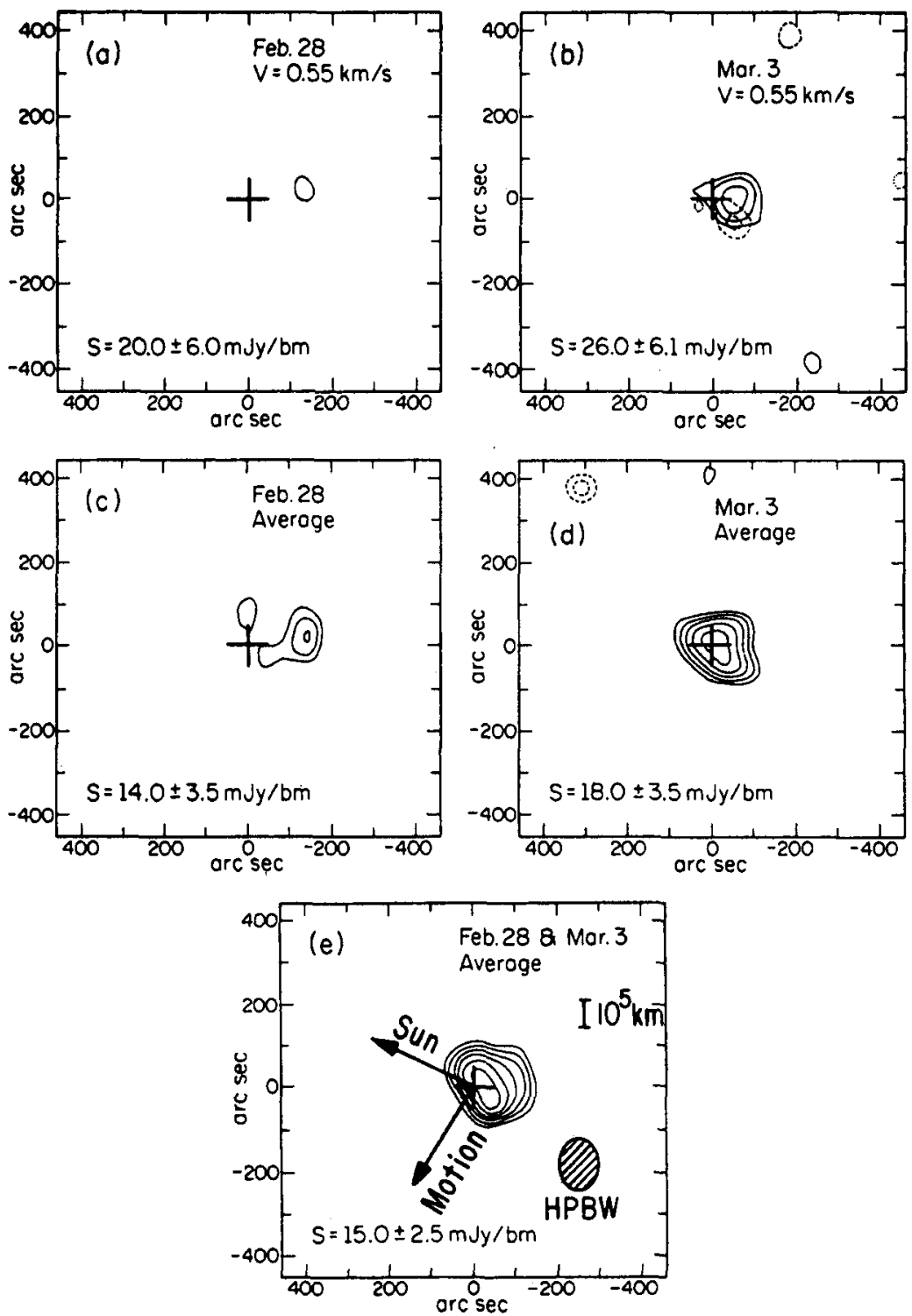

Fig. 8. Contour maps for the Comet Wilson data of February 28 and March 3. The presentation is like that in Fig. 7, except that here the dotted contours are for the channel one velocity step $(0.55 \mathrm{~km} / \mathrm{sec})$ more negative, and the dashed for one step more positive. Panels (c) and (d) are averages of the data for $v=0, v=+0.55$, and $v=+1.10 \mathrm{~km} / \mathrm{sec}$. 
to gas outflow alone. On February 6 and 7, 100 arcsec corresponded to a linear dimension of $1.86 \times 10^{5} \mathrm{~km}$ at Comet Wilson. Therefore, a relative motion of $1 \mathrm{~km} / \mathrm{sec}$ could cause a maximum angular motion of $46^{\prime \prime}$ per day. Some of the prominent differences are on a scale about 5 times larger than this. The middle panel of Fig. 7 shows an image of the data for each day averaged over three velocity channels; hence, this is the image that would result from an observation with high spatial but lower velocity resolution, $1.65 \mathrm{~km} / \mathrm{sec}$ rather than $0.55 \mathrm{~km} / \mathrm{sec}$. The emission pattern is more nearly round. An average of these images is shown in the bottom panel. This panel shows the effect of averaging over a larger velocity range $(1.65 \mathrm{~km} / \mathrm{sec})$, as well as a long integration time (2 days) - the image becomes even more symmetrical.

Figure 8 summarizes the data for February 28 and March 3 in the same format used in Fig. 7: the upper panel shows images for the channels with the maximum emission on each day indicated by the solid lines, and the contours of adjacent channels superimposed with dotted and dashed lines. The middle panel shows the average over three channels, and the bottom shows the average of two days' data. The February 28 data shows a marginal $(\sim 3 \sigma)$ signal at $v=+0.55 \mathrm{~km} / \mathrm{sec}$. The signal on March 3 is stronger; it peaks at $v=+0.55$ $\mathrm{km} / \mathrm{sec}$ and has comparable strength at $v=+1.10 \mathrm{~km} / \mathrm{sec}$. Just as was found from the data for February 6 and 7, each average makes the resulting image smoother and rounder.

\subsection{SPECTRAL LINE PROFILES}

From the VLA images, we can obtain spectral profiles by integrating for each velocity over patches on the sky surrounding all or part of the emission regions.

4.3.1 Comet Halley. Our line profile for the whole emission region on November 16 is shown in Fig. 9a. This profile is slightly asymmetric, with the center of the line at the comet's velocity and a FWHM of $1.8 \mathrm{~km} / \mathrm{sec}$, in good agreement with the results of Schloerb, Claussen and Tacconi-Garman (1987). Line profiles through individual blobs, however, show some asymmetries: although the peak flux density is always at a velocity of $0.0 \mathrm{~km} / \mathrm{sec}$, the centroid of a fitted Gaussian is slightly blueshifted for the blobs at the east side $(\sim 0.2$ $\mathrm{km} / \mathrm{sec}$ ), while the blobs to the south and west are redshifted. This implies that, in the reference frame of the comet, the blobs at the east move towards the sun, while those to the south and west in the opposite direction. In addition, the half power line width for the individual blobs is typically close to $1 \mathrm{~km} / \mathrm{sec}$, rather than $1.8 \mathrm{~km} / \mathrm{sec}$ as observed for the line profile integrated over the entire emission region.

The line profile for the whole emission region on January 4 is shown in Fig. $9 \mathrm{~b}$. On January 4 and 6 the peak flux density is redshifted to a velocity of $+0.55 \mathrm{~km} / \mathrm{sec}$. The profiles are also wider than in November, $\Delta v \simeq 2.2 \mathrm{~km} / \mathrm{sec}$. (This increase in width was also seen in the single dish data (see Table 2). Profiles through individual blobs generally resemble that in Fig. $9 \mathrm{~b}$ closely: the peak flux density is at $+0.55 \mathrm{~km} / \mathrm{sec}$ for all blobs, and they all show a general extension towards the red side of the spectrum.

4.3.2 Comet Wilson. In line profiles through the Comet Wilson images, the peak flux density is clearly displaced towards the blue in the early February data and towards the red on February 28 and March 3 (see also Table 2). These findings agree with the fact that the centroid of the $\mathrm{OH}$ brightness distribution on the sky (Figs. 7 and 8) is displaced towards the sun in early February, and in the opposite direction on February 28 and March 3. Figure 


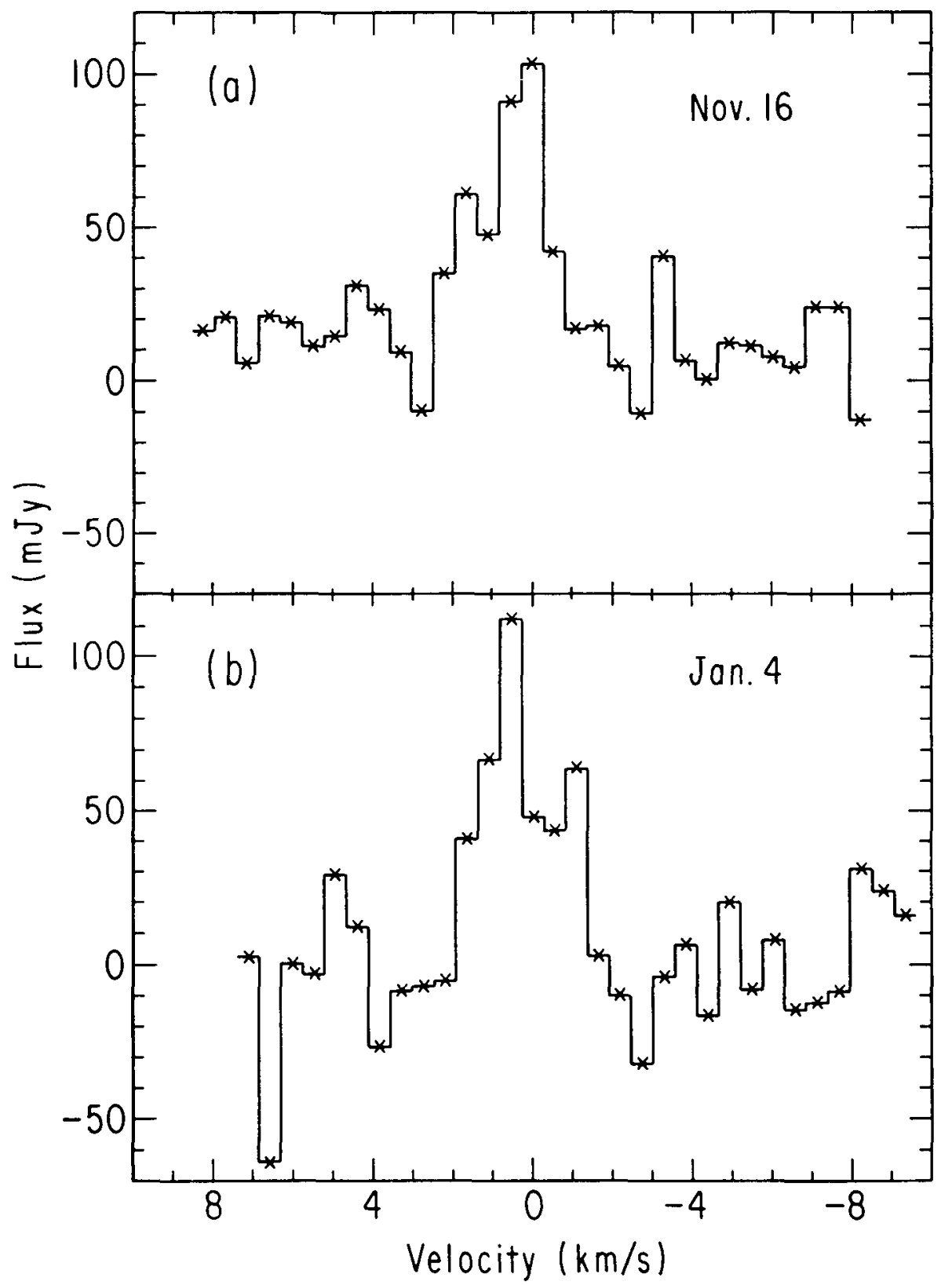

Fig. 9. Line profiles for Comet Halley, integrated over the entire emission region: (a) For November 16. (b) For January 4. These spectra and those in Fig. 10 can be compared with those obtained with a single-element radio telescope with a beamwidth of $\sim 15^{\prime}$. 
10 shows some examples of line profiles integrated over the entire emission region of Comet Wilson. Figure 10a is for February $6,10 \mathrm{~b}$ is for February 7 , and $10 \mathrm{c}$ is for the combined data from February 6 and 7 . The profiles for the individual days are narrow and peak at velocities of $-0.55 \mathrm{~km} / \mathrm{sec}$ on February 6 and $-1.10 \mathrm{~km} / \mathrm{sec}$ on February 7 . The average profile (Fig. 9c) demonstrates the effect of longer time averaging: it is broader. Profiles of smaller patches on separate days, while noisy, tend to be narrow and asymmetric in the sense that the blue side falls off more rapidly than the red side.

The line profiles of both Comets Halley and Wilson indicate that asymmetric line profiles may be more common than previously thought: it seems that long integration times with small single dishes (equivalent to integration over entire emission region) often tend to result in symmetric line profiles, while line profiles of the same comet integrated over a shorter time and/or observed with a larger antenna (integration over smaller areas on the sky) may show pronounced asymmetries.

\section{Discussion}

To interpret cometary data in terms of physical parameters of the comet, it is necessary to evaluate the inversion measure associated with the Swings and Greenstein effects. Figure 11 shows a graph of the inversion measure for the observing dates of comets Halley and Wilson, as a function of heliocentric velocity for the pumping models of Despois et al. (1981; solid line) and Schleicher and A'Hearn (1988; dashed line). Both curves were taken from Schloerb and Gerard (1985).

\subsection{TOTAL FLUX DENSITIES}

5.1.1 Comet Halley. Based upon the inversion measure, one would expect a monotonic increase in flux density by a factor of about 2 through November. In early January, one would expect a flux density 1/3 of that on November 13-16 (Schloerb and Gerard, 1985). Galt (1987) shows that the integrated flux density varied by as much as factors of 2-3 on time scales as short as one day. In November, the observed values were, on the average, below the predicted values, while during the first half of January, the observed flux densities increased from values below the predicted numbers to numbers well above the prediction. The total flux density received on January 4 with the 140-ft telescope in Green Bank was $\sim 50 \%$ that received on November 16 (Schloerb, Claussen and Tacconi-Garman, 1987). With the VLA we measured roughly equal amounts of emission on November 13-16 and January 4. As mentioned above, the flux density received with the VLA strongly depends upon the apparent size of the coma, and the small-scale structure of the emission region. The apparent size of the coma depends upon the comet-Earth distance, the lifetime of the $\mathrm{OH}$ molecules, and the production rate.

5.1.2 Comet Wilson. The inversion measure was largest on February 6, and decreased monotonically thereafter. Hence, one expects a larger $\mathrm{OH}$ flux density in early February than at the end of the month, which is in general agreement with the data (see Figs. 7 and 8). The flux densities shown in Table 2 indicate that the emission decreased by about $50 \%$ between February 6/7 and February 28/March 3. This is roughly in agreement with the prediction based upon the inversion measure from Fig. 11. The total flux density on March 

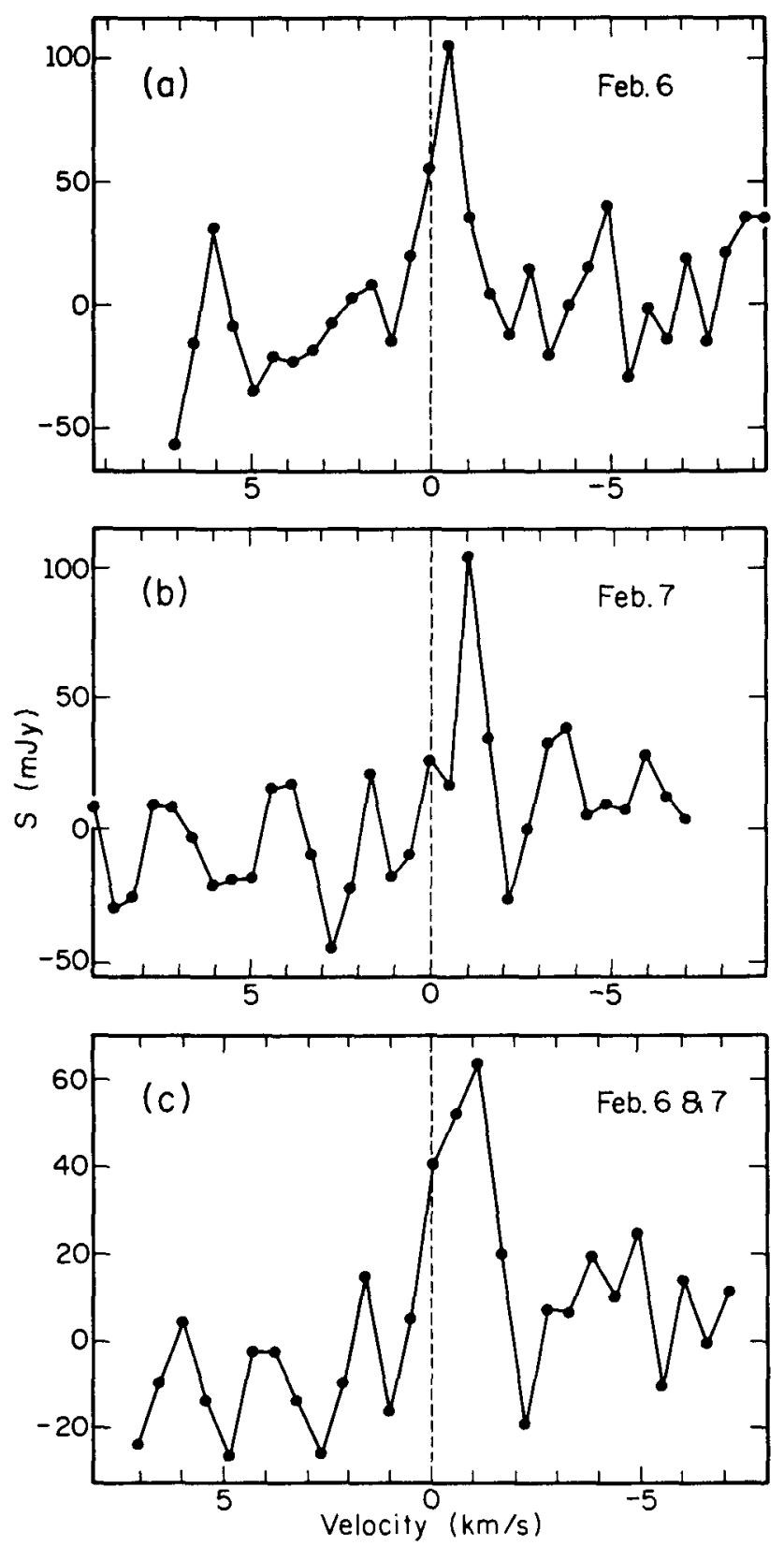

Fig. 10. Line profiles for Comet Wilson integrated over the entire emission region: (a) For February 6. (b) For February 7. (c) For combined data from February 6 and 7. 


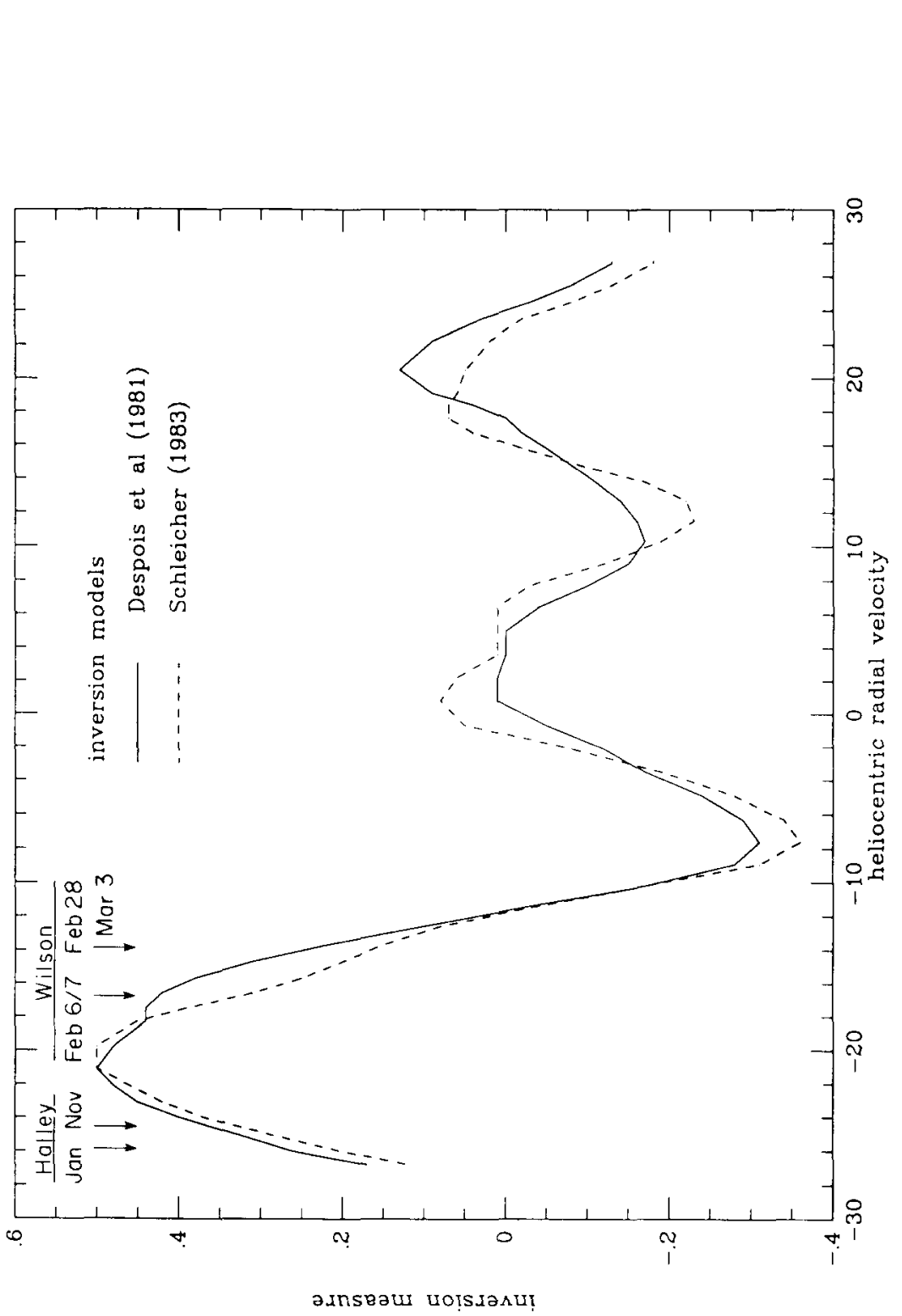


3, however, is expected to be $20 \%$ smaller than the value on February 28, but, in fact, a larger peak flux density is observed. Apparently, the production rate increased during the first few days of March. Hence, we may also see large daily fluctuations in Comet Wilson's production rate.

\subsection{PRODUCTION RATES}

Cometary observers usually publish their results in terms of an $\mathrm{OH}$ or $\mathrm{H}_{2} \mathrm{O}$ production rate, i.e., the number of $\mathrm{OH}$ or $\mathrm{H}_{2} \mathrm{O}$ molecules liberated by the comet per second of time. To calculate the production rate of a comet, however, one first needs to convert the observed flux density into the total number of $\mathrm{OH}$ molecules. Schloerb and Gerard (1985) gave the following expression for the total flux density emitted by the entire comet, integrated over all frequencies:

$$
S=\frac{A_{u l} i k T_{B G}}{4 \pi \Delta^{2}} \frac{2 F_{u}+1}{8} N_{O H}
$$

In eq. $1, A_{u l}$ is Einstein's coefficient, $i$ is the inversion measure, $T_{B G}$ is the background emission, $k$ is Boltzmann's constant, $F_{u}$ is the total angular momentum quantum number of the upper level of the transition, $N_{O H}$ is the total number of $\mathrm{OH}$ molecules in the ground state lambda doublet levels, and $\Delta$ is the geocentric distance to the comet. Thus the total number of ground state $\mathrm{OH}$ molecules is unambiguously defined with eq. 1 . The primary theoretical limitation of this equation is that $i$ is held constant: neither quenching (which reduces the value of, - and finally reverses the sign of - $i$ ) nor the Greenstein effect (which results in a value of $i$ depending on the location in the coma) are accounted for. The primary practical limitation is that only rarely is it possible to measure the total flux of a comet.

The measured flux density integrated over the line profile is often much less accurate than the peak flux density of the line. If one assumes a certain line profile, eq. 1 can be rewritten in terms of the peak flux density of the line. But estimates of the total number of $\mathrm{OH}$ molecules will be lower limits in most cases, since with both large single dishes and interferometric arrays, one measures only part of the flux density of the comet. To correct for the fraction of $\mathrm{OH}$ not directly observed, one needs to know the distribution of the molecules in the coma and the molecular outflow velocity. Usually a spherically symmetric outflow is assumed with either radial outfiow at a constant velocity (Haser model) or propagation of $\mathrm{OH}$ molecules in a random direction after photodissociation of radially outflowing $\mathrm{H}_{2} \mathrm{O}$ (a Monte Carlo approach). The $\mathrm{OH}$ brightness distribution of the gas, however, is very asymmetric as suggested by many of the observed line profiles (e.g., Bockelee-Morvan and Gerard, 1984), and as shown by the VLA observations summarized in this paper. So the assumption of symmetry is not met. Thus, although using a Haser or Monte Carlo model for the comet permits better estimates for the $\mathrm{OH}$ production rates, the assumption of symmetry can still introduce serious errors in the determination of $N_{O H}$.

The $\mathrm{OH}$ production rate is equal to the total number of $\mathrm{OH}$ molecules divided by the $\mathrm{OH}$ lifetime. Usually most molecules are in the ground state lambda doublet so this becomes $Q_{p}=N_{O H} / \tau_{O H}$. Unfortunately, the $\mathrm{OH}$ lifetime is not well known, and published values

differ by at least a factor of 2 (Schloerb and Gerard, 1985). Because $60-90 \%$ of the $\mathrm{H}_{2} \mathrm{O}$ photodissociates to $\mathrm{OH}$, and because $\mathrm{H}_{2} \mathrm{O}$ is assumed to be the parent molecule of $\mathrm{OH}$, one can estimate the production rate for water from the $\mathrm{OH}$ production rate. Given all the limitations above, however, one should not be surprised if this number does not agree with 
direct observations of water molecules.

\subsection{VELOCITY SHIFT OF THE LINE.}

5.3.1 Comet Halley. A rough comparison of Comet Halley's line shift and $\mathrm{OH}$ distribution with that expected from the Swings and Greenstein effects (Fig. 11) shows that for a 1 $\mathrm{km} / \mathrm{sec}$ outflow velocity for Comet Halley, the comet's anti-sunward side (along the cometsun line) ought to be brighter than the sunward side by a factor $\sim 1.6$ on November 13 and 16 , and by a factor of $\sim 3$ in January. Since integration along the line of sight will change these ratios, one really ought to compare the observations with calculations which model the entire cometary coma. A good estimate for the velocity shift, however, can be obtained from (Claussen and Schloerb, 1987):

$$
d v=v_{\text {out } f l o w}^{2} \frac{1}{3 i} \frac{d i}{d v} \cos (\omega)
$$

with $\omega$ the Sun-Comet-Earth angle, and $\frac{d i}{d v}$ the slope of the inversion measure. This expression gives an adequate description for a comet which has a symmetric outflow, provided that the slope $d i / d v$ remains constant throughout the coma. For both November and January we expect a red-shift in the velocity of $\sim 0.07 \times v_{\text {outflow }}$, which would not be detectable with our velocity resolution. As expected, no redshift has been detected for the line profile in November, but there is a large red-shift in January. This suggests anisotropic outgassing. 5.3.2 Comet Wilson. On February 6 and 7, as well as February 28 and March 3 Comet Wilson should have been brightest at the sunward side. The peak of the line profile is expected to be (immeasurably) blueshifted on all 4 days. Using the inversion model of Despois et al. (1981), we expect a blue-shift of $\sim-.01$ in early February and $\sim-.06$ in late February. Using Schleicher and A'Hearn's (1988) model, we find $\sim-.04$ and $\sim-.075$ respectively. While the sign of these predictions is in agreement with the early February data, even the sign is wrong for the data taken at the end of February.

The comparisons made above show that the $\mathrm{OH}$ emission is governed to the first order by the Swings and Greenstein effects, but that the appearance also can be drastically modified by the distribution of the gas. The data for Comet Wilson at the end of February show this most clearly: the side of the comet that ought to have been enhanced based upon the Greenstein effect, the sunward side, was not detected simply because there was not much gas at the sunward side of the comet during the VLA observations. This is not surprising, since the standard assumption of spherical gas outflow from comets has been shaken by the VEGA and the Giotto images showing gas and dust jets that may account for much of the gas production for Comet Halley (Keller et al., 1987). In addition, skewed, blueshifted profiles have been observed from time to time in single-element radio telescope studies of the 18- $\mathrm{cm} \mathrm{OH}$ lines from comets. These profiles could only be explained by invoking anisotropic outgassing approximately in the sunward direction (e.g., Bockelee-Morvan and Gerard, 1984). Further, on theoretical grounds, Combi (1987) has questioned the usual assumption that the gas flow around the comet becomes isotropic. 


\subsection{COMPARISON OF COMETS HALLEY AND WILSON}

It is interesting to compare the results from Comet Halley, an old periodic comet, and Comet Wilson, a new comet. Comet Wilson was three times as far from the Earth during the VLA observations as Comet Halley was in November 1985; hence the relative resolution for the two comets differs by a factor of three, which complicates a direct comparison. Fortunately, both comets were at approximately the same distance from the Sun when they were observed.

In the VLA observations of Comet Halley in November 1985 (de Pater, Palmer, and Snyder, 1986) and January 1986, the $\mathrm{OH}$ emission was primarily detected in a ring at 60,000 $\mathrm{km}$ from the nucleus. The VEGA spacecraft detected a transition region, the cometopause, at $\sim 10^{5} \mathrm{~km}$ from the nucleus (Gringauz et al.,1986). To compare the position of the cometopause with that of the clumps measured in the $\mathrm{OH}$ images, we need to know the comet's heliocentric distance at the times of observation, and a production rate of the comet. Since we need a consistent relative value for the production rates, we use the $\mathrm{OH}$ production rate values derived by Gerard et al. (1989) from their model 2. At the time of the VEGA observations, the heliocentric distance of the comet was $1 \mathrm{AU}$, and the $\mathrm{OH}$ production rate $\sim 10-20 \times 10^{28} \mathrm{~mol} / \mathrm{sec}$. The directly measured $\mathrm{H}_{2} \mathrm{O}$ production rate might seem preferable at this time, because the $\mathrm{OH}$ inversion measure was near zero, leading to large errors in the $\mathrm{OH}$ production rate. Because only the latter production rates are available during our November and January observations, we choose to use the $\mathrm{OH}$ production rate in March also. During the VLA observations, Halley's heliocentric distance was 1.73 and $0.93 \mathrm{AU}$ in November and January, respectively. The total $\mathrm{OH}$ production rate was $4.6 \times 10^{28}$ and $13.5 \times 10^{28} \mathrm{~mol} / \mathrm{sec}$ at these two times, respectively. By analogy with the magnetopause, we take the location of the cometopause to be such that the solar wind pressure and the pressure by the cometary plasma just balance. If the solar wind pressure is increased, the cometopause will move inward, approximately linearly with the increase in pressure (Schmidt and Wegman, 1982). If the pressure by the cometary plasma is increased, the cometopause will move outward. Since the heliocentric distance and $\mathrm{OH}$ production rates in March (VEGA encounter) and January were approximately the same, the cometopause should also be at $\sim 10^{5} \mathrm{~km}$ from the nucleus in January. In November, the heliocentric distance was larger by a factor of 1.86 ; thus the solar wind density and pressure were smaller by a factor of $\sim 3.5$ (this, of course, ignores time variations in the solar wind parameters). Since the $\mathrm{OH}$ production rate in November was about one-third of that in January, the cometopause must have been at a similar distance in both November and January. At both times, the ring of clumps is at $\sim 60,000 \mathrm{~km}$ from the nucleus, which is likely to be inside the cometopause. Hence, the appearance of the clumps may be the manifestation of the edge of the quenching region rather than the cometopause. Quenching will occur when the density is greater than a critical value, whose dimension scales as the square root of the total gas production rate. Since both the $\mathrm{OH}$ distribution and the distribution of other ions in the cometosphere are very irregular, the $\mathrm{OH}$ emission at this quenching edge may appear to be irregular.

The production rates of comets Halley and Wilson are similar; thus the quenching region is presumably of similar size, but, due to the three times lower resolution, the quenching region of Comet Wilson was not resolved. At best, the low-resolution image of Comet Halley can be compared with the images of Comet Wilson in this paper.

The most striking difference between the two comets is the apparent stability in the emission from Comet Halley, while the emission from Comet Wilson behaved very erratically, 
changing rapidly in position as well as in velocity. Interestingly enough, at UV wavelengths, just the opposite was observed: Comet Halley showed large fluctuations in the $\mathrm{OH}$ production rate, while Comet Wilson appeared quiescent, with an $\mathrm{OH}$ production rate that showed a smooth dependence on heliocentric distance (Festou et al., 1986; Roettger et al., 1989). The $\mathrm{H}_{2} \mathrm{O}$ production rate determined by Larson et al. (1989) from IR measurements showed no pronounced temporal variability, but the observed cometocentric shift and line widths suggest high expansion velocities. However, it should be noted that both the IR and UV observations of Comet Wilson were made a few months later than the radio observations, when the comet was nearer perihelion. Moreover, compared with the world-wide effort given to Comet Halley, Comet Wilson was rather sparsely observed. Therefore, neither the radio, IR, nor UV data permit as strong statements about variability as could be made for Comet Halley.

Another interesting difference between the two comets is that the small scale structures in the $\mathrm{OH}$ emission from Comet Halley seems confined to a region a few times $10^{5} \mathrm{~km}$ in size, while the emission from Comet Wilson shows up in sporadic blobs, with variable intensities and velocities, at distances as far as $10^{6} \mathrm{~km}$ from the center. As was mentioned above, these distances are too large to be explained by spherical outflow of parent $\mathrm{H}_{2} \mathrm{O}$ / daughter $\mathrm{OH}$ molecules from the nucleus. It might appear that $\mathrm{OH}$ excitation variations are responsible, but at $10^{6} \mathrm{~km}$ from the nucleus, the gas density is sufficiently low that the UV pumping will be much faster than any other excitation rate. Therefore, the only reasonable possibility left is that $\mathrm{OH}$ is produced near these spots by a secondary source of molecules. For a new comet, which is being subjected to the stresses of passage through the inner solar system for the first time, some fragmentation of the aggregated cometesimals in the outer mantle or outer frosting may be expected (see Whipple, 1989). Therefore, the outer, relatively transient blobs observed in the $\mathrm{OH}$ emission from Comet Wilson may be associated with the disintegration of the outer frosting and possibly with the fragmentation and ejection of cometesimals from the nucleus. A rough estimate of the size of these cometesimals can be obtained from the flux density in the individual blobs. We estimate the sizes of the order of 50-100 m.

\section{Conclusions}

In this paper we presented an overview of observations obtained with the VLA, and in particular we discussed the $\mathrm{OH}$ observations. A brief discussion of radio interferometry and the "detectability" of a comet by the VLA was presented in Section 2. It has been shown that the VLA receives over $90 \%$ of the comet's flux density if the size of the emission region is less than $\sim 3^{\prime}$ (for a Gaussian source); the source will still be visible if its size is 10 12 ', but the flux density will be decreased to $\sim 30 \%$ of the total. If the source consists of discrete small features, the VLA has no trouble detecting them, as long as they are within the primary beam of the telescope.

In Section 3 we described all the cometary observations attempted to date with the VLA. Successful observations were obtained of the $\mathrm{OH}$ emission from comets Halley and Wilson, and of $\mathrm{H}_{2} \mathrm{CO}$ emission from comets Halley and Machholz. It was found that the formaldehyde must come from an extended source in the cometary coma, rather than just the nucleus. The fractional abundance of $\mathrm{H}_{2} \mathrm{CO}$ in Comet Halley was $\sim 1.5 \%$. 
It was further pointed out in Section 3 that the VLA is a very powerful instrument for detection of line transitions from parent molecules. We may note here that large millimeter arrays would be even more powerful; in the near future such experiments can be attempted with the expanded millimeter array of the BIMA consortium* ( $~ 6$ dishes) at the Hat Creek Radio Observatory.

Images of comets at the $1667-\mathrm{MHz}$ transition of $\mathrm{OH}$ have been obtained for Comet Halley, an old periodic comet, and Comet Wilson, a comet that entered the inner solar system for the first time. The emission from Comet Halley seems confined to a region a few times $10^{5}$ $\mathrm{km}$ in size, while the emission from Comet Wilson shows up in sporadic blobs at distances as far as $10^{6} \mathrm{~km}$ from the center. The latter emission varies significantly in intensity as well as velocity. This behavior may be associated with the disintegration of the outer frosting associated with new comets and possibly with the fragmentation and ejection of cometesimals from the nucleus. The emission from Comet Halley displayed a remarkable stability. The emission shows up in discrete blobs at the approximate distance of the cometopause, which is coincidently the approximate distance to the edge of the quenching region. This suggests that the emission at these distances may be controlled by collisional processes.

We further showed in this paper that asymmetric cometary $\mathrm{OH}$ line profiles may be more common than previously thought. Lengthening the integration time and lowering the velocity resolution give the image the smooth, round shape predicted for an isotropic outflow of gas. In addition, spectral profiles become more symmetric after long integration times and after integrations over larger spatial regions. Images and spectral profiles from observations integrated over short time intervals may have erratic profiles and brightness distributions. It should be clear that it will be very important in the future to carefully coordinate radio, optical, and UV observations to avoid some of the ambiguities that are present in the existing data.

Acknowledgements. I.d.P. acknowledges support from NSF grants AST-8514896 and AST-8900156 to the University of California, Berkeley, and the Alfred P. Sloan foundation. Both L.E.S. and P.P. acknowledge support from NASA grant no. NAGW-1131 to the University of Illinois.

*Operated by the University of California at Berkeley, the University of Illinois, and the University of Maryland, with support from the National Science Foundation. 


\section{References}

Altenhoff, W.J., W. Bartla, W.K. Huchtmeier, J. Schmidt, P. Stumpff, and M. Walmsley, Radio Observations of Comet 1983d, Astron. Astrophys., 125, L19-L22, 1983.

Altenhoff, W.J., W.K. Huchtmeier, J. Schmidt, J.P. Schraml, and C. Thum, Radio Continuum Observations of Comet Halley, Astron. Astrophys., 164, 227-230, 1986.

Ananthakrishnan, S., P.K. Manoharan, and V.R. Venugopal, Scintillations of Four Radio Sources Occulted by the Plasma Tail of Comet Halley in, Cometary Radio Astronomy, eds. W. M. Irvine, F. P. Schloerb, and L. Tacconi-Garman, NRAO Workshop No. 17, Green Bank, W. Virginia, 35-39, 1987.

Bracewell, R., Image Reconstruction in Radio Astronomy in, Image Reconstruction from Projections, Springer-Verlag, ed. G.T. Herman, Berlin, Heidelberg, New York, 81-105, 1979.

Bockelee-Morvan, D., and E. Gerard, Radio Observations of the Hydroxyl Radical in Comets With High Spectral Resolution. Kinematics and Asymmetries of the $\mathrm{OH}$ Coma in C/Meier (1978XXI), C/Bradfield (1979X), and C/ Austin (1982g);Astron. Astrophys. 131, 111-122, 1984.

Clark, B.G., An Efficient Implementation of the Algorithm "CLEAN", Astron. Astrophys., 89, 377, 1980.

Claussen, M.J., and F.P. Schloerb, Radio OH Observations of P/Halley With the NRAO 43-m Telescope in, Cometary Radio Astronomy, eds. W. M. Irvine, F. P. Schloerb, and L. Tacconi-Garman, NRAO Workshop No. 17, Green Bank, W. Viriginia, 135-141, 1987.

Crovisier, J., and F.P. Schloerb, The Study of Comets at Radio Wavelengths, this volume, 1990 .

Combes, M., V.I. Moroz, J.F. Crifo, J.M. Lamarre, J. Charra, N.F. Sanko, A. Soufflot, J.P. Bibring, S. Cazes, N. Coron, J. Crovisier, C. Emerich, T. Encrenaz, R. Gispert, A.V. Grigoryev, G. Guyot, V.A. Krasnapolsky, Yu.V. Nikolsky, and F. Rocard, Infrared sounding of comet Halley from VEGA 1, Nature, 321, 266-268, 1986.

Combi, M. R., Sources of Cometary Radicals and Their Jets: Gases or Grains, Icarus, 71, 178-191, 1987.

Combi, M.R., and A.H. Delsemme, Neutral Cometary Atmospheres. I. An Average Random Walk Model for Photodissociation in Comets, Astrophys. J., 237, 633-640, 1980.

Cornwell, T., Braun, R., Deconvolution in, Synthesis Imaging in Radio Astronomy, eds. R.A. Perley, F.R. Schwab, A.H. Bridle, Astronomical Society of the Pacific, NRAO Workshop no. 21; 167-183, 1989.

de Pater, I. and W.-H. Ip, A Radio Source Occultation Experiment by Comet Austin (1982g) with Unusual Results, Astrophys. J., 283, 895-904, 1984.

de Pater, I., P. Palmer, and L.E. Snyder, The Brightness Distribution of OH Around Comet Halley, Astrophys. J. Lett., 304, L33-L36, 1986.

de Pater, I., P. Palmer, L.E. Snyder, and W.-H. Ip, VLA Observations of Comet Halley: The Brightness Distribution of $\mathrm{OH}$ Around the Comet in, Proceedings of the 20th International ESLAB Symposium on the Exploration of Halley's Comet, Heidelberg, Germany, 409-412, Oct. 27-31, 1986. 
de Pater, I., C.M. Wade, H.L.F. Houpis, and P. Palmer, The Non-detection of Continuum Radiation from Comet IRAS-Araki-Alcock at 2- to 6- $\mathrm{cm}$ Wavelengths and its Implication on the Icy Grain Halo Theory, Icarus, 62, 349-359, 1985.

Despois, D., E. Gerard, J. Crovisier, and I. Kazes, The OH Radical in Comets: Observation and Analysis of the Hyperfine Microwave Transitions at $1667 \mathrm{MHz}$ and $1665 \mathrm{MHz}$, Astron. Astrophys., 99, 320-340, 1981.

Eberhardt, P., D. Krankowsky, W. Schulte, U. Dolder, P. Lammerzahl, J.J. Berthelier, J. Woweries, U. Stubbemann, R.R. Hodges, J.H. Hoffman, and J.M. Illiano, The CO and $\mathrm{N}_{2}$ Abundance in Comet P/Halley, Astron. Astrophys., 187, 481-484, 1987.

Festou, M.C., The Density Distribution of Neutral Compounds in Cometary Atmospheres. I. Models and Equations, Astron. Astrophys., 95, 69-79, 1981a.

Festou, M.C., The Density Distribution of Neutral Compounds in Cometary Atmospheres. II. Production Rate and Lifetime of $\mathrm{OH}$ Radicals in Comet Kobayashi-Berger-Milon (1975 IX), Astron. Astrophys., 96, 52-57, 1981b.

Festou, M. C., P.D. Feldman, M.F. A'Hearn, C. Arpigny, C.B. Cosmovici, A.C. Danks, L.A. McFadden, R. Gilmozzi, P. Patriarchi, G.P. Tozzi, M.K. Wallis, and H.A. Weaver, IUE Observations of Comet Halley During the Vega and Giotto Encounters, Nature, 321, 361-363, 1986.

Galt, J., Monitoring the $1.667 \mathrm{GHz}$ Line in Comet Halley, Astron. J., 93, 747-752, 1987.

Gerard, E., The OH Radio Lines in Comets: A Review, in, Cometary Radio Astronomy, eds. W.M. Irvine, F.P. Schloerb, and L. Tacconi-Garman, NRAO workshop no. 17, Green Bank, WV, 91-98, 1987.

Gerard, E., D. Bockelee-Morvan, G. Bourgois, P. Colom, and J. Crovisier, Observations of $\mathrm{OH}$ at $18 \mathrm{~cm}$ in Comets P/Halley 1982i, P/Giacobini-Zinner 1984e, C/Hartly-Good 19851 and C/Thiele $1985 \mathrm{~m}$, in , Cometary Radio Astronomy, eds. W. M. Irvine, F. P. Schloerb, and L. Tacconi-Garman, NRAO Workshop No. 17, Green Bank, WV, $125-133,1987$.

Gerard, E., D. Bockelee-Morvan, G. Bourgois, P. Colom, and J. Crovisier, Observations of the OH Radio Lines in Comet P/Halley 1986 III, Astron. Astrophys. Suppl. Series, 77, 379-410, 1989.

Gringauz, K. I., T.I. Gombosi, A.P. Remizov, I. Apathy, I. Szemerey, M.I. Verigin, L.I. Denchikova, A.V. Dyachkov, E. Keppler, I.N. Klimenko, A.K. Richter, A.J. Somogyi, K. Szego, S. Szendro, M. Tatrallyay, A. Varga, and G.A. Vladimirova, First in Situ Plasma and Neutral Gas Measurements at Comet Halley, Nature, 321, 282-285, 1986.

Hoban, S., and S. Baum, A VLA Search for 2-cm Continuum Radiation from Comet Halley, Icarus, 70, 264-268, 1987.

Hobbs, R.W., J.C. Brandt, and S.P. Maran, Microwave Radiation From Comet West, 1975n, Astrophys. J., 218, 573-578, 1977.

Hobbs, R.W., S.P. Maran, J.C. Brandt, W.J. Webster, Jr., and K.S. Krishna Swamy, Microwave Continuum Radiation from Comet Kohoutek 1973f: Emission from the Icy Grain Halo, Astrophys. J., 201, 749-755, 1975.

Högbom, J.A., Aperture Synthesis With a Non-regular Distribution of Interferometer Baselines, Astron. Astrophys. Suppl., 15, 417-426, 1974.

Ip, W.-H., An Overview of Gas Phenomena in Comet Halley, Adv. Space Res., 5, 233-245, 1985.

Jackson, W. M., T. Clark and B. Donn, Radio Detection of $\mathrm{H}_{2} \mathrm{O}$ in Comet Bradfield 
(1974b), in, The Study of Comets, eds. by B. Donn, M. Mumma, W. Jackson, M. A'Hearn, and R. Harrington, NASA SP-393, Washington, D.C., 272-280, 1976.

Keller, H. U., W.A. Delamere, W.F. Huebner, H.J. Reitsema, H.U. Schmidt, F.L. Whipple, K. Wilhelm, W. Curdt, R. Kramm, N. Thomas, C. Arpigny, C. Barbieri, R,M. Bonnet, S. Cazes, M. Coradini, C.B. Cosmovici, D.W. Hughes, C. Jamar, D. Malaise, K. Schmidt, W.K.H. Schmidt, and P. Seige, Comet P/Halley's Nucleus and its Activity, Astron. Astrophys., 187, 807-823, 1987.

Krankowsky, D., The Composition of Comets, this volume 1990.

Kraus, J.D., Radio Astronomy, Cygnus Quasar Books, Powell, Ohio, 1986.

Larson, H.P., H.A. Weaver, M.J. Mumma, and S. Drapatz, Airborne Infrared Spectroscopy of Comet Wilson (19861) and Comparisons with Comet Halley, Astrophys. J., 338, 1106-1114, 1989.

Palmer, P., I. de Pater, and L.E. Snyder, VLA Observations of the OH Emission from Comet Wilson (1986I): the Value of High Resolution in Both Spatial and Velocity Coordinates, Astron. J., 97, 1791-1797, 1989.

Perley, R.A., Schwab, F.R., Bridle, A.H., Synthesis Imaging in Radio Astronomy, Astronomical Society of the Pacific, NRAO Workshop no. 21, 1989.

Roettger, E. E., P.D. Feldman, M.F. A'Hearn, M.C. Festou, L.A. McFadden, and R. Gilmozzi, IUE Observations of the Evolution of Comet Wilson (19861): Comparison with P/Halley, Icarus, 80, 303-314, 1989.

Rolhfs, K., Tools of Radio Astronomy, Springer-Verlag, Berlin Heidelberg, 1986.

Rots, A., Special Purpose Image Display Devices and Techniques at the National Radio Astronomy Observatory, Computers in Physics, vol. 3, no. 6, 41-46, 1989.

Schenewerk, M., P. Palmer, L.E. Snyder, and I. de Pater, VLA Limits for Comets Austin (1982 VI) and P/Crommelin (1983n): Evidence for a Diffuse OH Halo, Astron. J., 92, 166-170, 1986.

Schleicher, D.G., The Fluorescence of Cometary $O H$ and $C N$, Ph.D. Dissertation, Univ. of Maryland, 1983.

Schleicher, D.G., and M.F. A'Hearn, The Fluorescence of Cometary OH, Astrophys. J., 331, 1058-1077, 1988.

Schloerb, F.P., Collisional Quenching of Cometary Emission in the 18 Centimeter OH Transitions, Astrophys. J., 332, 524-530, 1988.

Schloerb, F.P., and E. Gerard, Models of Cometary Emission in the 18-cm OH Transitions: The Predicted Behavior of Comet Halley, Astron. J., 90, 1117-1135, 1985.

Schloerb, F.P., M.J. Claussen, and L. Tacconi-Garman, OH Radio Observations of Comet P/Halley, Astron. Astrophys., 187, 469-474, 1987.

Schmidt, H.U., and R. Wegmann, Plasma Flow and Magnetic Fields in, Comets, ed. L.L. Wilkening, University of Arizona Press, Tucson, Arizona, 538-560, 1982.

Snyder, L.E., A Review of Radio Observations of Comets, Icarus, 51, 1-24, 1982.

Snyder, L.E., The $18 \mathrm{~cm}$ OH Lines in Comets: Preparation for Halley, Astron. J., 91, 163-170, 1986.

Snyder, L.E., P. Palmer, and I. de Pater, Radio Detection of Formaldehyde Emission from Comet Halley, Astron. J., 97, 246-253, 1989a.

Snyder, L.E., P. Palmer, and I. de Pater, Observations of Formaldehyde in Comet Machholz (1988j), Icarus, in press, 1989b.

Snyder, L.E., P. Palmer, and C.M. Wade, An Upper Limit to the Microwave Continuum 
Radiation from Comet Austin (1982g), Astrophys. J. Lett., 269, L21-23, 1983.

Sramek, R.A., Map Plane - $u v$ Plane Relationships, in Proc. NRAO-VLA workshop held in Socorro, New Mexico, June 21-25, 2.1-2.13, 1982.

Sramek, R.A., and F.R. Schwab, Imaging, in Synthesis Imaging in Radio Astronomy, ed. R.A. Perley, F.R. Schwab, A.H. Bridle, NRAO Workshop no. 21, Astronomical Society of the Pacific, 117-138, 1989.

Thompson, A.R., Moran, J.M., Swenson, G.W.Jr., Interferometry and Synthesis in Radio Astronomy, John Wiley and Sons, New York, 1986.

Weaver, H.A., M.J. Mumma, and H.P. Larson, Infrared Investigation of Water in Comet P/Halley, Astron. Astrophys, 187, 411-418, 1987.

Whipple, F. L., Comets in the Space Age, Astrophys. J., 341, 1-15, 1989.

Wright, C.S., and G.J. Nelson, Comet Plasma Densities Deduced from Refraction of Occulted Radio Sources, Icarus, 38, 123-135, 1979. 